Running head: "SOCIAL DILEMMAS IN RIDESHARING"

\title{
When a Few Undermine the Whole: \\ A Class of Social Dilemmas in Ridesharing
}

Amnon Rapoport*, Hang Qi, Vincent Mak, and Eyran J. Gisches

August 08, 2019

Amnon Rapoport, Eller College of Management, University of Arizona, 1130 East Helen Street, Tucson, Arizona 85721, USA, Phone: +(1) 520.621.9325, Fax: +(1) 520.621.4171, Email: amnon@u.arizona.edu

Hang Qi, Institute for Advanced Studies in Finance and Economics, Hubei University of Economics, Wuhan, P.R. China, Email: qihang@tju.edu.cn

Vincent Mak, Cambridge Judge Business School, University of Cambridge, Trumpington Street,

Cambridge CB2 1AG, United Kingdom, Phone: +44(0)1223-764295, Email: v.mak@jbs.cam.ac.uk

Eyran J. Gisches, Eller College of Management, University of Arizona, 1130 East Helen Street, Tucson, Arizona 85721, USA, Phone: +(1) 520.621.4546, Email: eyran@email.arizona.edu

* Correspondence author: Amnon Rapoport. All four co-authors have contributed equally.

Acknowledgement: This research has been supported by NSF Grant SES-1418923 awarded to the University of Nevada at Las Vegas and the University of Arizona. 
When a Few Undermine the Whole:

\title{
A Class of Social Dilemmas in Ridesharing
}

\begin{abstract}
We investigate a class of social dilemmas that arise when a heterogeneous group of agents potentially benefit from a joint enterprise such as ridesharing. Participation in the enterprise incurs positive externalities to other participants; social welfare is maximized with full participation. However, if some agents find it a dominant strategy to opt out, then the potential benefit from the enterprise will decrease, leading to more members opting out. This iterated disincentivizing effect could result in massive welfare losses. We construct a game-theoretical model to implement these social dilemmas and report experimental evidence for their existence and welfare impact.
\end{abstract}

Keywords Social dilemmas; Ridesharing; Cost-sharing; Route choice in traffic networks; Heterogeneous agents; Experiment

JEL Classification C72, C91, D80, R41 


\section{When a Few Undermine the Whole:}

\section{A Class of Social Dilemmas in Ridesharing}

\section{Highlights}

- A class of social dilemmas in ridesharing and similar joint enterprises

- A few individuals might find it always preferable to opt out

- Benefit of the initiative decreases as a result, leading to further opting out

- Could result in massive losses in social welfare

- Theoretical and experimental evidence for their existence and welfare impact 


\section{When a Few Undermine the Whole: \\ A Class of Social Dilemmas in Ridesharing}

\section{Introduction}

New communication technologies have led recently to the blossoming of ridesharing public services such as Uber and Lyft. These operators provide service for areas that are not served well, or not served at all by public transportation, and often act as a transit system feeder. They attract customers largely for the lower transportation cost they provide compared with other available options. Reduction of transportation cost is critically dependent on the number of the participating customers: the higher the number of participants in ridesharing, the lower the shared cost, and the more benefit the customers reap from the arrangement.

In this study, we investigate a class of social dilemmas that arise in ridesharing and similar joint enterprises with positive externalities. The decision situation that we study involves independent network users choosing the same transportation mode and sharing the travel cost equally. In the social dilemmas that emerge from the situation, a majority of the users would have benefitted from ridesharing as long as there was a high level of participation because of the associated positive externalities. Yet, the user population could still end up with a high level of non-participation, or even complete non-participation. The reasons are as follows: some (perhaps only a small minority) of the individuals might find it always preferable to opt out from ridesharing because their private costs are very low compared to the others. If that happens, then the potential number of participants decreases, so that the potential benefit from the joint initiative also decreases, leading to further opting out by more users. This iterated disincentivizing effect - in fact, a manifestation of the iterated elimination of dominated strategies - might then result in a low participation level, or even complete non-participation, and massive welfare losses. 
Similar outcomes may appear in other scenarios that are different from ridesharing. The common feature of these scenarios is that they involve participation in a joint enterprise (e.g., construction of a community swimming pool), so that the enterprise is more beneficial to group welfare the higher the number of participants. If some individuals in the group prefer to opt out from the joint enterprise, then the potential number of participants decreases and, consequently, more group members opt out. The iterated disincentivizing effect could again result in massive losses of group benefit. Schelling (1978, p.102-110)'s “dying seminar” model has related insights. In his model, people attend a seminar only if a critical mass of other people are expected to attend, and different people may have different critical masses. In our model as well, different players have different critical masses for ridesharing to be preferable to private transport. Some players may have critical masses that are well below the maximum potential number of ridesharing participants; however, both our theoretical and empirical findings show that even those players could eventually opt out of ridesharing. Another comparable model is Dixit (2003)'s club game. The model involves similar positive externalities as ours, while the analysis focuses on how the presence of one player, to whom joining the club is a dominant strategy, can ultimately lead to all players joining - an outcome that might be socially suboptimal. Breitmoser, Tan, and Zizzo (2014)'s experimental test of an extensive-form version of Dixit's model shows considerable adherence to equilibrium predictions, but also evidence of bounded rationality. Here, we look at a relatable setting in the context of ridesharing but focus on "flip-side" social dilemmas in which no players join the "club" of ridesharing even though all might have benefitted from it.

After reviewing relevant literature, in the following sections we construct a ridesharing model to demonstrate the social dilemmas. The model is structured as a traffic network game that presents group members a choice between traveling alone on a route with an individual private 
cost that is heterogeneous among the group members or, alternatively, traveling with other group members and sharing the joint travel cost equally. To account for the individual choices, we compare to each other two outcomes: an equilibrium solution derived from non-cooperative game theory, which is suboptimal for the group, and another non-equilibrium outcome that maximizes the group social welfare. We then report a behavioral test of the model by means of an experimental game with two between-group conditions. One condition has a unique corner-solution equilibrium with no players choosing ridesharing; the other has a unique interior equilibrium with half of the players choosing ridesharing. For both conditions, we obtain consistent experimental evidence for the existence of the theorized social dilemmas as well as their welfare impact.

Our study makes several contributions to previous research on social dilemmas. First, we propose a class of social dilemmas that are quite different in nature from well-known major social dilemmas (see e.g., Mak and Rapoport 2013). Social dilemmas in public goods games, including classic versions with symmetric payoff functions and no-contribution equilibria (see Ledyard 1995), or modified versions with asymmetric payoff functions and interior equilibria (e.g., McGinty and Milam 2013), are typically driven by free riding on non-excludable public goods. In our model, the social dilemmas are induced by players who opt out of an excludable joint initiative. Consider also the standard threshold public goods game (e.g., Croson and Marks 2000). The game has more than one feasible outcome according to game theory analysis using the concept of Nash equilibrium; one of these equilibrium outcomes leads to provision of the public good and maximizes the welfare of the group. The social dilemma occurs where, behaviorally, individuals fail to coordinate on that optimal equilibrium outcome but instead converge to inferior equilibria (see Van Huyck et al. 1990, 1991, for similar issues in general coordination games, and Liu, Mak, and Rapoport 2015). A crucial point is the dynamics of mutual influence among individuals upon 
repeated interactions, which is often the key to whether the group converges to either an optimal or an inferior outcome. As such, the inferior outcome might only emerge gradually over the course of the experiment. By contrast, in the scenarios investigated in the present study, the only equilibrium outcomes are suboptimal, and the social dilemmas occur with certainty.

Secondly, through our experiment we offer behavioral evidence for the theorized social dilemmas in terms of both their existence and drastic welfare impact. In our experiment, it appears that the iterated disincentivizing effect had played out as part of the strategic decision-making process of the participants before they made their decisions. As a result, in either of the two experimental conditions, inferior group outcomes appeared from the very start of the session and did not improve as the session progressed.

Thirdly, the social dilemmas emerge from a route choice setting in a traffic network with a ridesharing option. It adds behavioral perspectives and empirical evidence to theoretical studies on cost sharing (see, e.g. Moulin 2003), and similar situations could occur in more general instances of joint enterprises. As such, our work also contributes to research on interactive behavior by illustrating a new class of social dilemmas in the context of directed networks.

\section{Experimental Literature Review}

Previous literature on social dilemmas includes both theoretical and experimental studies and stretches back several decades. The relevant points of connections with the present study are as discussed above. Reviews of the literature appear in Croson and Marks (2000), Chaudhuri (2011), Dawes (1980), Ledyard, (1995) Mak and Rapoport (2013), Messick and Brewer (1983), Van Lange et al. (2013), Zelmer (2003), and others. Here, we provide a brief review of the experimental literature related to the other major aspect of our work, namely, route choice in directed networks - a literature that has been growing rapidly in recent years and, therefore, might be less familiar. 
There is a rich theoretical literature on choice of routes in directed networks in which traversal across any edge (link) could only be in one direction (see, e.g., Nisan et al. 2007; Roughgarden 2005). The theoretical literature has instigated much experimental research in which choices of route are examined in controlled laboratory settings under strategic uncertainty. Routes are typically chosen by members of small groups who submit their decisions independently. Their decisions are decentralized, and they are typically homogenous in incentive terms. This stream of research might be traced back to the seminal paper by Pigou (1920) on directed networks with only two routes that connect a single origin and a single destination. One of the routes has a fixed travel cost and is consequently not susceptible to congestion, and the other has a variable travel cost that increases linearly in the number of its users.

Pigou's canonical example was examined in later experiments on route choice by Iida, Akiyama, and Uchida (1992), Selten et al. (2007), Knorr, Chmura, and Schreckenberg (2014), and Mak, Gisches, and Rapoport (2015) among others. The results of these studies support equilibrium predictions for aggregate, but not individual, choice behavior. Other route choice experiments that involve complexities such as exogenous risks ( $\mathrm{Lu}$ et al. 2014) reveal more significant deviations from equilibrium predictions; these results have prompted researchers such as Dixit and DenantBoemont (2014) and Wijayaratna et al. (2017) to interpret the deviations with modified equilibrium concepts. A related topic is the study of departure time in transportation networks with bottleneck congestion (see, e.g., Arnott, de Palma, and Lindsey 1990 for a theoretical treatment). Gabuthy, Neveu, and Denenat-Boemont (2006) find significant deviations from equilibrium in their experiment with a simple bottleneck. Daniel, Gisches, and Rapoport (2009)'s experiment, which involves a more complex two-bottleneck setting, reported results that were more consistent with equilibrium predictions at an aggregate level, probably because the experiment involved as 
many as 50 rounds of play - compared with Gabuthy et al. 2006's 15 rounds of play - to facilitate learning.

Pigou (1920) also has given rise to experimental research on several traffic paradoxes in congestible networks. Morgan et al. (2009) and subsequently Hartman (2012) conducted experimental studies of the Pigou-Knight-Downs paradox, which states that expanding the capacity of a road segment with the intention to reduce congestion may not reduce travel cost because it may elicit new demand. Denant-Boémont and Hammische (2010) and later Dechenaux et al. (2014) reported studies of the Downs-Thomson paradox (Downs 1962; Thomson 1977). This paradox states that improving the capacity of one of the two routes in a network may lead to more commuters switching from the public to the private route, thereby increasing travel costs of all the commuters. Rapoport et al. (2006, 2009, 2014) and Gisches and Rapoport (2012) reported several studies of the Braess paradox (Braess 1968) stating that, paradoxically, adding a single link that connects two parallel and congestible routes in a directed network from a common origin to a common destination may increase the travel time of the network users. The joint outcome of these studies provides strong support for equilibrium predictions when the architecture of the network is enriched, group size assumes different values, the stage game is iterated sufficiently many times, and the group members are homogenous. In these studies, there is no evidence in support of outcomes that maximize group welfare as opposed to equilibrium outcomes that are inferior in social welfare.

Like these previous papers, the present study focuses on directed networks in which users choose their routes with no central authority to manage the flow. In sharp contrast to most of them, which focus on congestible games, we focus on ridesharing in which route choices may impose positive externalities on other users, since the benefits of choosing the ridesharing route are an 
increasing function of the number of the network users choosing the same route; see Anshelevich et al. (2004) for an example of a theoretical treatment, and Furuhata et al. (2013) for a literature review. We take forward the modelling analysis of previous works and present here a class of related social dilemmas with supporting experimental evidence.

We conclude this section with a brief review of a previous experimental study that is more directly related to the present study. Liu et al. (2015) reports the results of an experiment on choice of routes between a single origin and a single destination. The cost of one of the three routes called the private route - was fixed in one of their two experimental conditions, and drawn randomly for each player in the second condition from a commonly known distribution. The travel cost on either of the two additional routes, called the public routes, was shared equally among players choosing the same route. In the first condition, the players were homogenous with equal private cost, and in the second condition they were not. Whereas the network game in Liu et al. (2015) has three pure-strategy equilibria, the game in the present study has only a single equilibrium. Importantly, the best equilibrium outcome in Liu et al. maximizes group welfare. Therefore, their study is about whether players could coordinate their decisions to maximize welfare. In contrast, the equilibrium in each of the two conditions of the present study is inferior to the group welfare-maximizing outcome, so that our study is not about coordination to attain an optimal equilibrium.

\section{Theoretical Analysis}

\subsection{The Cost-sharing Game}

Consider the following scenario faced by a group of $n$ persons, who happen to fly together to attend a conference, football match, public concert, or a wedding ceremony. After their flight has landed, the travelers disembark from the airplane and each of them needs to choose between 
ordering a taxicab to her hotel (e.g., to freshen up, register to the hotel, etc.) or ridesharing on a van directly to the conference/football match/concert hall/wedding hall. The ridesharing option has a limited capacity that is $n$. The choice between the private (taxicab) and public (ridesharing) modes of transportation is made individually and independently.

The cost of taking a taxicab varies from one person to another depending on the location of the person's hotel; some are close to the airport and some are far away; see Figure 1 for a schematic representation of the distances. Meanwhile, persons who choose ridesharing would be splitting the travel cost equally. It might have been presumed that these travelers, with asymmetric private (taxicab) costs, could have freely bargained among themselves to achieve a cooperative game-theoretic allocation that is asymmetric (see e.g., Moulin 2003, Section 5.1). Here, we assume that time and other social or market constraints deprive the travelers from the opportunity of extended negotiation, so that ridesharing travelers share the cost equally as a rule. We also assume that the public mode of transportation travels along a special lane (e.g., a carpool or highoccupancy vehicle lane) that incurs a different total cost from any of the private route costs.

- Insert Figure 1 about here -

The cost-sharing model in this study is a stylization of the above scenario. In the model, a group of $n$ players could choose sharing their travel costs in the fashion of ridesharing. Specifically, the network has two modes of transportation that are modeled by $n+1$ directed routes. Of these are $n$ routes, each one exclusive to each of the $n$ players, with fixed travel costs that differ widely from one player to another. We refer to these routes as private routes, and denote each of them by $(O$, $\left.D_{j}\right), j=1,2, \ldots, n$. The network also includes an additional route, called the public route, which may accommodate all the $n$ players. The players who choose this route share its travel cost, denoted as $c$, equally. Denote the number of players who choose the public route in the game as $i(i=0,1$, 
$\ldots, n)$. Then the travel cost of a player on the public route is simply $c / i$. Regardless of the values

of the (fixed) travel costs on their respective private routes, the more players choosing to travel on the public route (i.e., the larger is $i$ ), the lower their individual travel costs. Routes are chosen independently with complete information about the cost structure. Since each private route is exclusive to some player, we use the same indices for private route and player. Hence, denote a player by $j, j=1,2, \ldots n$, and her travel cost on her exclusive private route by $c_{j}, c_{j}>0$. With no loss of generality, order the players such that $c_{j}>c_{j+1}>0$ for all $j$.

\subsection{The Cost Structures for the Experiment}

In order to illustrate the social dilemma, we focus on a scenario with a group of $n=10$ players with a particular set of private route costs as shown in the top panel of Table 1 . These costs follow the formula $c_{j}=(100 / j)-7$ with each result rounded down to the nearest integer. For example, the private route cost is 93 for player $j=1,43$ for player $j=2$, and so on down to $c_{j}=3$ for player $j=$ 10; the players are highly heterogeneous in that the highest and lowest private route costs differ from each other by a factor of 31 . If all the 10 players choose their private routes, then the total travel cost is $c_{1}+c_{2}+\ldots+c_{10}=221$ (see top panel of Table 1). The same private route cost structure was used throughout the experimental sessions.

The public, cost-sharing vehicle is assumed to have a capacity of $n=10$. For the total travel cost on the public, cost-sharing route, we focus on two levels that are used in our experiment to define two between-subjects experimental conditions. Specifically (see the right-hand column of the bottom panel of Table 1), the total travel cost on the public route is $c=c_{\mathrm{H}}=120$ for Condition $\mathrm{H}$ (for "high"), and $c=c_{\mathrm{L}}=60$ for Condition L (for "low"). In the experiment, we rounded down the shared cost to the nearest integer whenever needed, so that the shared costs are as listed in 
Table 1. In both conditions, the sum of the private route costs across all the 10 players (i.e., 221) exceeds the total cost of the public route: $c_{1}+c_{2}+\ldots+c_{10}>c_{g}$ for $g \in\{\mathrm{L}, \mathrm{H}\}$.

- Insert Table 1 about here -

Inspection of Table 1 shows that each of the 10 players in Condition $\mathrm{H}$ would prefer choosing her private route rather than being the only player who chooses cost-sharing: $c_{j}<c_{\mathrm{H}}$ for $j \in\{1,2, \ldots, 10\}$. Moreover, the five players with the lowest private travel costs (players 6 through 10) in Condition $H$ will always prefer the private route as $c_{j}<c_{H} / 10$ for $j \in\{6,7,8,9,10\}$. In contrast, player $j=1$ in Condition $\mathrm{L}$, who has the highest private travel cost, would prefer choosing the public route as $c_{1}>c_{\mathrm{L}}$, whereas players 8,9 , and 10 in Condition $\mathrm{L}$, who have the lowest private costs, would prefer traveling on the private route, as $c_{j}<c_{\mathrm{L}} / 10$. To continue this line of thinking, the middle panel of Table 1 lists the critical mass for each player in each condition: the critical mass is defined as the minimum number of players other than that player, who have chosen the public route in order for the public route to be at least as preferred as the private route.

\subsection{Equilibrium Analysis; the Social Dilemmas}

To generate theoretical predictions for the model, we conduct an analysis focused on purestrategy Nash equilibrium solutions. In these solutions, $i^{*}$ players choose the public route (ridesharing) and the remaining $n-i^{*}$ players choose the private route (taxicab), where $i^{*}$ is an integer in $\{0,1,2 \ldots n\}$. Recall that we rank order the indices $j$ of the players so that $c_{j}>c_{j+1}$ for $j$ $=1,2, \ldots n$-1. If, in equilibrium, player $j>1$ chooses the public route, then player $j-1$ must find the public route preferable to the private route, since that player could only face a higher cost for the private route than player $j$. Thus, in an interior equilibrium with $i *$ players choosing the public route where $n>i^{*}>0$, those players must be player $1,2, \ldots, i^{*}$, while player $i^{*+1}, i^{*+2}, \ldots, n$ 
must be choosing their private routes. The feasibility conditions for the pure-strategy Nash equilibrium must be:

$$
c_{i^{*}} \geq c / i^{*} \text { and } c /\left(i^{*}+1\right) \geq c^{* *+1} .
$$

For the equilibrium in which $i^{*}=0$ (all the players choose the private routes), the feasibility condition must be $c \geq c_{1}$ and $c / n \geq c_{n}$. For the equilibrium in which $i=n$ (all the players choose the public route), the feasibility condition must be $c_{n} \geq c / n$.

As is apparent from the above results, a social dilemma occurs when $c_{1}+c_{2}+\ldots+c_{n}>c$ (so that all choosing the public route maximizes group welfare), but $i^{*}<n$. Indeed, this is what happens in the two experimental conditions with $n=10$.

We first read off the parameters in Table 1 to ascertain which equilibrium is feasible for each condition. The only feasibility condition that is satisfied in Condition $\mathrm{H}$ is $c_{\mathrm{H}} \geq c_{1}$ and $c_{g} / 10$ $\geq c_{10}$. Hence, the unique pure-strategy equilibrium in Condition $\mathrm{H}$ is for all the 10 players to choose their respective private routes. The only feasibility condition that is satisfied in Condition $\mathrm{L}$ is $c_{5}$ $\geq c_{\mathrm{L}} / 5 \geq c_{6}$. Hence, the unique pure-strategy equilibrium in Condition $\mathrm{L}$ is an interior one with players $1,2,3,4$, and 5 choosing the public route and the remaining five players choosing their respective private routes. Equivalently, we can arrive at the same conclusions by appreciating that the critical mass (see Table 1) of a player is never lower than the player's role index in Condition $\mathrm{H}$, and is lower than the player's role index in Condition $\mathrm{L}$ only for players $1,2,3,4$, and 5 .

It is important to note that in both conditions, the total cost for all players would be minimized if all players were to choose the public route, because $c_{1}+c_{2}+\ldots+c_{10}>c_{g}$ for $g \in\{\mathrm{L}$, $\mathrm{H}$ \}. However, this outcome is not the equilibrium in either of the conditions, as $i^{*}<10$ in both conditions. That is, both equilibria are not welfare maximizing for the group, resulting in a social dilemma in either condition. The upshot is that, in equilibrium in Condition $\mathrm{H}$, the total cost to the 
group (221) is 1.84 times the welfare-maximizing total cost of 120 , if all players choose the public route - a substantial "price of anarchy" as discussed in Mak and Rapoport (2013). In the equilibrium in Condition L, the total cost to the group (88) is 1.47 times the welfare-maximizing total cost of 60 .

It is also important to note that the games in both conditions are dominance solvable, and the equilibria may be constructed by elimination of dominated strategies. For example, in Condition H, players 6 to 10 find the private route a strictly dominant strategy, since their private costs are lower than the minimum public route cost of 12 even when all the players choose that route. Therefore, players 1 to 5 (who would have preferred the public route had all players chosen it) could reason that at most only five players might choose the public route, meaning that the cost of the public route would be at least 24 , which is higher than the private route costs of players 4 and 5. This reasoning leads to an iterated elimination of dominated strategies, a disincentivizing effect in which players 1 to 3 could now reason that players 4 and 5 would also not choose the public route. Thus, at most three players might choose the public route with a minimum cost of 40, where player 3 would find it preferable to choose her private route as well. Two more iterations lead to even player 1, with the highest private cost, to opt out of choosing the public route. Thus, no player in Condition $\mathrm{H}$ chooses the public route in equilibrium. These are iterations in reasoning, which might happen as part of the players' strategic thinking before anyone commits to any choice.

Similarly, in Condition L, players 1 to 7 might have benefitted from ridesharing had all the players chosen it. In contrast, players 8, 9, 10 find that their private route is a strictly dominant strategy. In turn, this leads to an iterated elimination of dominated strategies, a disincentivizing effect that results in players 6 and 7 refraining from the public route. As a result, only half of the 
population choose the public route in equilibrium. Again, these iterations in reasoning could happen before anyone commits to any choice, leading to an instantaneous social dilemma.

\section{The Experiment}

In this section, we report an experimental game designed to simulate in a controlled laboratory environment the two conditions discussed in the previous section. The overall objective of the experiment is to gauge the descriptive validity of the social dilemmas and their welfare impact.

It is not a forgone conclusion that the social dilemmas, in the form of convergence of decisions towards the respective equilibria, would occur empirically. The participants in Conditions $\mathrm{H}$ and $\mathrm{L}$ were instructed that the stage game would be repeated in the experiment 80 times with random assignment of player roles upon each repetition. This provided a strong incentive for the participants to choose the public route collectively and thereby achieve a socially optimal low mean cost across iterations of the stage game. As we report below, the participants seemed to ignore this incentive and quickly converged to the socially inferior equilibrium.

\subsection{Participants}

Two hundred participants in approximately equal proportions of males and females took part in the experiment. The participants were mostly undergraduate students, who volunteered to take part in a decision-making experiment for payoff contingent on their performance. One hundred participants were assigned randomly to each of the two conditions (Conditions $\mathrm{H}$ and L) in a between-subjects design. For each condition, there were 10 sessions (groups) of 10 players each, which differed from each other only in the total travel cost of the public route.

\subsection{Design and Procedure}

Upon entering the laboratory, the participants were seated randomly in separate cubicles; communication between them was not possible. Each participant was handed written instructions 
(see Online Appendix A), which she read at her own pace. The instructions presented a network diagram with 11 routes in a "diamond" form. The "diamond" format was simply a means to visualize the cost structures for the participants. If the travel cost on the private route is broken into two parts, namely, $c_{j}$ for the first arm of the link and 0 for the second arm, then the network in Figure 1 and the network displayed in the instructions (Online Appendix A) are topologically equivalent. In particular, the two networks are also equivalent in terms of the strategic interactions and the implied social dilemmas.

The instructions explained the cost structure in detail, and the computation of the payoff was illustrated with a numerical example. The participants were informed that the game would be repeated for 80 identical rounds, and that on each round they would be randomly assigned a player role. They also were informed that the payoff for each round (in experimental tokens) would be determined by subtracting their travel cost from a reward of 50 tokens for reaching their destination. The value of the reward was deliberately set sufficiently low to ensure sensitivity to travel costs (the higher the reward, the less important the perceived differences between the costs), and yet sufficiently high to reduce the likelihood of multiple rounds with negative payoffs. Player roles were assigned randomly subject to the constraint that every participant was assigned each of the 10 player roles an equal number (i.e., eight) of times in the experimental session.

At the beginning of each round, each participant was presented with a computer screen showing her player role $j$, and the tabulated private route costs of all the $n$ players. The screen also had two clickable buttons, one corresponding to each route, for the participant to submit her decision. At the end of the round, the program displayed a second computer screen that presented to each participant her player role, the cost structure, the route that she had chosen, her payoff for the round, the number of players who had chosen the private route, and the complementary number 
of the players who had chosen the public route (see Online Appendix A). Each participant could access at any time a personal history screen, which listed her previous route choices with their associated payoffs.

At the end of the session, each participant was paid in cash a $\$ 5.00$ participation bonus plus her cumulative earnings according to the exchange rate $\$ 1.00=110$ tokens. The mean participant payment was $\$ 24.7$ and $\$ 34.7$ for Conditions $\mathrm{H}$ and L, respectively, including the $\$ 5.00$ bonus. Each session lasted approximately one hour on average.

\section{Results}

- Insert Table 2 and Figure 2 about here -

\subsection{Preliminary Analysis}

Recall that in the equilibrium in Condition $\mathrm{H}$, all the 10 group members choose the private route; while in Condition L, players 1-5 choose the public route, and players 6-10 choose the private route. Note also that because the cost-sharing game in the experiment was repeated 80 times with player roles randomly re-assigned on each round, the participants had an incentive to deviate from equilibrium and choose the public route to maximize the mean individual payoff as well as the group welfare in the session. However, this strategy was not widely adopted by any group in the experiment. In both conditions, participants started out far from the social optimum and quickly converged around the theoretical, equilibrium outcomes, which were grossly inferior in terms of group welfare.

Table 2 displays for each group separately the mean number or choice frequencies of the participants traversing the public route in each of the four blocks of 20 rounds in the experimental sessions. Overall means by group and block, as well as the overall per player travel cost in each group, are also listed in the table. Lastly, the equilibrium prediction and social optimum (i.e., when the group would have minimized their total travel cost) are also listed for comparison. 


\subsubsection{Non-significant Deviations from Equilibrium Prediction}

Overall, the groups in the two conditions converged quickly towards their respective and very different equilibria. With group as the unit of analysis, the choice frequencies of the public route in both conditions were significantly different even from round 1. The mean choice frequencies of the public route in round 1 were $2.70(1.16)$ in Condition $H$ and $4.00(1.25)$ in Condition L, respectively (standard deviations in parentheses). The difference between these two means is statistically significant according to a $t$-test comparison with group as the unit of analysis $(t(18)=2.41, p=0.027)$. Subsequently, in every block in Condition $\mathrm{L}$ the overall mean choice frequencies of the public route (see Table 2) rose from around the equilibrium prediction of 5 ( $p>$ 0.05 in $t$-test comparisons for deviations from equilibrium in blocks 1 and 2 , with group as the unit of analysis) to a higher level of 5.53 on average in both blocks 3 and 4 . The latter mean choice frequency was numerically just above the equilibrium prediction but was statistically significantly different from it $(p<0.05$ in $t$-test comparisons for blocks 3 and 4 also with group as the unit of analysis). See Section 5.2 for further discussion on the reasons behind this deviation.

Similar standard statistical tests for deviations from equilibrium do not apply to Condition $\mathrm{H}$ because the equilibrium choice frequency of the public route is the floor value of zero. However, the convergence in this condition is clearly evident in Table 2 as well as Figure 2, which exhibits the mean choice frequencies across all 10 groups of the public route by round in both conditions.

Overall, the mean choice frequencies of the public route in Conditions $\mathrm{H}$ and $\mathrm{L}$ differ significantly: 0.39 in Condition H vs. 5.36 in Condition L, $t(18)=23.0, p<0.01$, with group as the unit of analysis.

\subsubsection{Significant Deviations from Social Optimum; Welfare Impact}


As shown in Table 2, the choice frequencies of the public route are clearly different from the social optimum where all players choose the ridesharing option. As the social optimum benchmarks are all ceiling (for choice frequencies of the public route) or floor (for per player travel cost) values, standard statistical tests do not apply. However, the gap between observed values and the social optimum are distinctly wide, especially given the standard deviations.

Importantly, the 10 groups in both conditions suffered severe welfare losses relative to their potential social optimum. The overall individual mean travel cost in Condition $\mathrm{H}$ was 22.94, which was almost double the social optimum value of 12 ; likewise, the overall mean travel cost of a player in Condition L was 9.18, which was 1.53 times the social optimum value of 6 . In other words, the participants in our experiment left a lot of money on the table, thereby suffering substantial empirical prices of anarchy (Mak and Rapoport 2013) of 1.91 and 1.53 in Conditions $\mathrm{H}$ and $\mathrm{L}$, respectively.

\subsubsection{Within-session Evolution}

Table 2 and Figure 2 also show that the participants in both conditions approached the equilibrium rather quickly and their route choices overall stabilizing just above the equilibrium level. As indicated in Table 2(a) (column 13), in Condition $H$ there was some learning over the first half (i.e., the first two blocks or Rounds 1-40) of the session. Afterwards, the choice frequencies of the public route stabilized just above the equilibrium prediction (with floor value of zero). There was typically some noisy attempts by a few individuals to pull the group out of the equilibrium, especially in Group H10 (see Figure A.1 in Online Appendix B); however, such attempts were largely unsuccessful in causing large-scale deviations from equilibrium. Similarly, in Condition L there was some learning over the first half of the session after which fluctuations persisted until the end of the session, with choice frequencies of the public route converging 
slightly above the equilibrium level of 5 . The observations reported in this subsection are consistent with supplemental Figures A.1 and A.2 in Online Appendix B, which exhibit the choice frequencies of the public route in each group in Conditions $\mathrm{H}$ and $\mathrm{L}$.

\subsection{Deviations from Equilibrium; Analysis by Player Role}

On average, the deviations from equilibrium in Condition $\mathrm{H}$ accounted for only 3.88 percent of choice observations (31.0 out of 800 decisions per group on average). When calculated separately for the two halves of the session (Rounds 1-40 and Rounds 41-80), the mean deviation score dropped sharply from 6.55 percent in the first half to 1.20 percent in the second half. This is borne out in the top panel of Figure 3, which exhibits the number of deviations from equilibrium by player role for the first and second half of the session separately. Player roles are exhibited on the horizontal axis, and the frequencies of deviations on the vertical axis. The sharp drop in deviations is apparent in the panel. This is especially drastic among players $1-5$, who might have lowered their travel costs by choosing the public route had the other players chose that route as well. When the participants were playing in these roles, they were much more likely to deviate from equilibrium, but only in the first half of the session; in the second half of the session they all had learned to follow equilibrium in whichever player role they were in. A within-subjects 10 (Player roles) $\times 2$ (first vs. second half of the session) ANOVA of the number of deviations, with group as the unit of analysis, yields a significant main effect over the two halves of the session $(F(1,9)=29.14, p<0.01)$, together with main effect in player role as well as interaction effect, both at $p<0.01$. These learning effects are all consistent with Figure 3 as well as the within-session evolution analysis. 
Deviations from equilibrium in Condition L were much higher at 11.31 percent of the choice observations ( 90.5 out of 800 per group on average). Deviations in this condition persisted throughout the session but with a slight decline as the session progressed: the percentage of route choices that deviated from equilibrium was 13.25 percent in the first half and 9.38 percent in the second half of the session. This is also borne out in the bottom panel of Figure 3. A within-subjects 10 (player roles) $\times 2$ (first vs. second half of the session) ANOVA of the number of deviations, with group as the unit of analysis, yields a significant main effect over the two halves of the sessions $(F(1,9)=10.28, p=.011)$.

In Condition L, deviations from equilibrium could be in the form of choosing the private route when the equilibrium choice was the public route (player roles 1-5), or choosing the public route when the equilibrium choice was the private route (player roles 6-10). As is evident in Figure 3, the deviations among player roles 1-5 dropped sharply across the two halves, but the deviations from equilibrium among player roles 6-10 were not very different between the two halves. Correspondingly, in the preceding ANOVA, there was a significant main effect for player role $(F(9,81)=10.54, p<.01)$ as well as an interaction effect $(F(9,81)=4.39, p<.01)$. The persistent imbalance in deviations by player roles results in the observed positive deviations in number of players choosing the public route even in those rounds, which is consistent with the within-session evolution analysis.

\subsubsection{Further Discussion on Deviations in Condition L and Inequity Aversion}

Why did some level of deviations from equilibrium persist throughout the session among player roles 6-10 in Condition L? Note that, by definition, those players had no individual incentives to deviate unilaterally from equilibrium by choosing the public route. In fact, even the welfare-maximizing outcome held little attraction (if at all) for these player roles. Specifically, 
player roles 6 and 7 could improve their travel costs by 3 and 1 tokens (relative to their respective private route costs) if all choose the public route; player roles 8-10 could not improve at all, and in fact should choose the private route as a dominant strategy. Yet experimental participants in these player roles chose the public route with consistent frequencies. Note that these results are partially within-subjects in nature because of the random reassignment of roles from round to round; that is, it seems that some participants deviated from equilibrium to different extents in different roles, and persistently so through the session. A simple explanation based on a lack of equilibrium reasoning may not be able to account for such asymmetry in deviations. The observations thus call for an explanation beyond attempts at self-interest maximization.

One possibility is that participants cared about relative payoffs in the form of inequity aversion (Fehr and Schmidt 1999). In the present experimental game, the private route costs are highly heterogeneous. As shown in Table 1, the cost for player role 1 is 93 tokens, for player role 4 it is 18 tokens, and for player role 10 it is 3 tokens. The corresponding ratios are 31:1 for player roles 1 and 10, and 6:1 for player roles 4 and 10. These are enormous differences. Thus, a player who chooses the private route might experience significant disadvantageous inequality if his/her player role incurs a relatively high travel cost on the private route, such as for player role 1 or 2. Likewise, a player who chooses the private route might experience advantageous inequality if his/her player role incurs a relative low private route cost, such as for player role 9 or 10 . On the other hand, when all the players choose the public route, there is no inequality; even when only a few group members choose the public route, those choosing it can experience complete equality among themselves.

As such, inequity aversion might motivate a player to choose the public route when equilibrium prescribes choosing the private route. This possibility applies to any player in 
Condition $\mathrm{H}$ and player roles 6-10 in Condition L. In Condition H, as is evident from our data analysis, if deviations had been partially motivated by inequity aversion (in addition to attempts to reduce travel costs), the deviations declined as the session progressed. Meanwhile, in Condition L, deviations from player roles 6-10 persisted throughout the session, and might have arisen from aversion to advantageous inequality. That is, if participants in player roles 6-10 were averse to advantageous inequality, they might be willing to choose the public route for the sake of equality even if that meant sacrificing some amount of self-interest; after all, the amount to be sacrificed was typically not considerable (see also Section 5.3.1). Our data are consistent with this possibility.

\subsection{Individual-level Analysis}

We next report individual-level analysis that offers additional insights into the choice behavior in the experiment. In each of the 10 groups in Condition $\mathrm{H}, 85$ percent of the participants deviated not more than five times, and most of these deviations took place at the beginning of the session. In contrast, in Condition L the range of individual deviations (between 0 and 43) was more pronounced. Nevertheless, the distributions are highly skewed by outliers; in every one of the 10 groups in Condition $\mathrm{H}$ at least half of the participants deviated not more than 10 times. These observations are supplemented by Figure A.3 in Online Appendix B, which exhibits box-andwhisker plots of the distribution of individual players' number of deviations from equilibrium choice.

- Insert Table 3 about here -

\subsubsection{Why was Convergence around Equilibrium Prevalent?}

We next compute the correlations, separately for each condition, between a player's total travel cost in the session and his/her total number of deviations from equilibrium route choice. The correlation coefficients are 0.70 and 0.65 for Conditions $\mathrm{H}$ and L, respectively; both values are 
positive and statistically significant $(p<0.01)$. These results imply a high payoff sacrifice when not following equilibrium route choice. We further confirm the results by linear regressions of individual travel cost on individual number of deviations from equilibrium over all rounds. We conducted a regression for each condition separately with participant as the unit of analysis. To account for correlations among data points, we used the GEE (General Estimating Equations, Liang and Zeger 1986) approach through the GENMOD procedure in SAS. The GEE approach does not require a full specification of the underlying correlational structure of the data; it yields estimations by iteratively solving a system of equations based on quasi-likelihood distributional assumptions. The results are listed in Table 3, which shows that the relevant regression coefficients are as follows (standard error in parentheses): Condition H: 26.47 (1.72); Condition L: 8.17 (1.95).

Even if the participants had realized that they should maximize overall payoffs throughout the session by repeatedly choosing the public route, such attempts must have proved to be very costly, and as a result were adopted infrequently. For example, in Condition $\mathrm{H}$, the average cost in equilibrium is 22.1 (see Table 2(a)). A participant could do better than that through always choosing the public route only if at least six participants in total consistently deviated from equilibrium by choosing the public route. For Condition L, the average cost in equilibrium is 8.8 ; a participant could do better than that through always choosing the public route only if at least seven participants in total consistently chose the public route, which is two more than the equilibrium choice frequency. Without communication and binding agreements, our participants were unable to achieve that. These observations help explaining why convergence to the deficient equilibrium, rather than the social optimum, was so prevalent in our experiment.

As discussed in the previous subsection, deviations from equilibrium in Condition L could be in the form of taking the private route when the equilibrium prescribes choosing the ridesharing 
public route (player roles 1-5) or vice versa (players roles 6-10). Therefore, we refined our analysis by conducting for Condition $\mathrm{L}$ a linear regression of individual travel cost over rounds when the participant was in player roles $1-5$, on individual number of deviations from equilibrium in those same rounds, with the participant as the unit of analysis and with standard errors clustered by groups. We then conducted a similar regression for the players in roles 6-10. The results yield consistent positive coefficients for the relevant number of deviations variable (see Table 3): for player roles 1-5, the coefficient (standard error in parentheses) is 20.25 (2.56); for player roles 610 , the coefficient is $3.27(0.35)$. We also carried out a series of similar regression analysis for each role separately. We find that travel cost is significantly and positively related to the relevant number of deviations for all the roles $(p<0.01)$ except for role 6 , where the coefficient is 0.55 with standard error 0.29 , and is marginally significantly different from zero $(p=0.068)$. This result may be explained by the fact that, for player role 6 , the travel costs of the private and public routes differ by only a single payoff unit when the rest of the group members are playing according to equilibrium (see Table 1).

Lastly, recall that the overall estimated coefficient is 8.17 , a value that is closer to the coefficient for player roles 6-10. This is because a predominant 65.75 percent (595 out of 905) of the deviations in Condition L were by participants in player roles 6-10, which is understandable given that the potential additional cost in deviation (risking public instead of the prescribed private route) for those player roles is much lower than that for player roles 1-5 (see the previous subsection). The same observation explains why the coefficient for player roles 6-10 is noticeably lower than for player roles 1-5. It also helps explaining why deviations from player roles 6-10 could persist throughout the session.

\section{Concluding Remarks}


We investigate a class of social dilemmas that arise in decision scenarios involving a joint enterprise such as ridesharing. In these dilemmas, a group of individuals potentially benefit from participating in the enterprise, such as sharing the transportation cost in a ridesharing arrangement. The benefit of the enterprise increases with the total number of participants, and social welfare is maximized if there is full participation by all the group members. However, if a few of the group members find it always preferable to opt out, then the potential benefit from the joint initiative will also decrease, leading to more group members opting out. This iterated disincentivizing effect - a manifestation of the iterated elimination of dominated strategies - might result in massive welfare losses. As discussed earlier, this class of social dilemmas are inherently different from well-known major social dilemmas, such as the classic public goods and threshold public goods games.

We construct a model to demonstrate the social dilemmas theoretically as well as experimentally. For the latter purpose, we conducted a computer-controlled experiment using a stylized traffic network with a group of 10 participants who were assigned heterogeneous roles. In our design, the players are asked to choose repeatedly between either a private route with a fixed travel cost or a public, ridesharing route with which the travel cost is shared equally with other players. The cost structures across the two experimental conditions only differ from each other in the total travel cost on the public route. In both conditions, group members are assigned widely dispersed costs for their own exclusive private routes. Correspondingly, the theoretical predictions, which are not welfare maximizing for the group, are for all the players in the high-cost condition and half of the players in the low-cost condition to choose the private route. In both conditions, the outcome that maximizes group welfare is for all the group members to choose the public route and share the travel cost equally. 
Our stylized model is inspired by a story about people who happen to fly together to attend a public event such as a conference or a football match (Section 3.1). Our experimental design involves repeated play of a stage game that replicates this scenario in the laboratory, with random reassignment of private route costs (i.e., player roles) from round to round. The random role assignment might be motivated by scenarios such as researchers in the same field attending the same conference every year but incurring different private route costs each time - which could be due to changes in conference locations in the same city and personal reasons such as budget constraints, staying in the same hotel with friends, and sharing hotel rooms. Our design essentially assumes that, while private route costs can change from year to year, the distribution of these costs among the players is approximately the same. More generally, this repeated scenario captures the case where the same individuals periodically choosing between paying for varying individual usage costs and sharing a fixed pooled cost. Further illustrative examples include a group of friends who periodically choose between paying individually for their mobile data usages or sharing a plan; or a neighborhood of residents who periodically choose between contracting garbage collection services individually based on volume of garbage (which is a function of family size) or sharing these services under a fixed monthly fee.

Our experimental results support the descriptive validity of the theorized social dilemmas in these scenarios and exhibit their drastic welfare impact. It seems that the participants in the experiment readily and quickly followed strategic reasoning that led them towards equilibrium choices - a general conclusion that is only qualified by slight deviations in Condition L that might have been driven by inequity aversion (see Section 5.2). As a result, inferior outcomes emerged at the very start of the experimental session in both conditions and did not improve as the session progressed. It might have been expected that random reassignment of player roles from round to 
round could rationalize long-term collusion to maximize mean session payoff, so that every participant would choose the public route in every round. This is especially reasonable for Condition $\mathrm{L}$ as even its lower private route costs are not highly superior to the equilibrium cost on the public route (Table 1). However, this was not the case in the experiment, and the outcome serves as another example of the validity of the equilibrium prediction when large groups (with no ability to communicate effectively) are concerned. Perhaps random reassignment of player roles also meant that repeating the same signal to collude on two or more consecutive rounds was difficult. Further research is warranted on facilitating tacit collusion in this class of social dilemmas, such as through communication, rewards, and other means of norm setting.

The social dilemmas that we study have potentially important implications. From consumer ridesharing to management of collaborative teams, our work highlights the importance of motivating individuals who would find it least beneficial to partake in the relevant joint initiatives. Not only their own non-participation will impact group welfare and team performance; the iterative disincentivizing effect on other individuals could lead to much higher levels of non-participation with severe financial consequences. Even when high participation level in cost sharing or collaboration is obviously beneficial to the group, it could take only a few individuals' nonparticipation to undermine the whole enterprise. If those few individuals might be persuaded that a critical number of other individuals would also participate, and that differential benefits to collaboration might be reversed in future interactions, it would be possible to appeal to their forward-looking thinking and self-control (see, e.g., Kocher et al. 2017) in order to rally consistent support for collaboration. As our experiment demonstrates, if such initiatives might ever be efficacious they should be best carried out with very consistent and emphatic communications or the enterprise would still falter. 
A major future research direction is concerned with how the social dilemma might be averted by structural changes in the model design. For example, is it possible for a policy maker to cost/subsidize the system in a way to mitigate the social dilemma and its inefficiency? Specifically, the policy maker might tax private route transportation to an extent that even the lowest private route cost is at least higher than the public route cost when all choose the public route (i.e., $c_{n}{ }^{\prime}>c / n$, where $c_{n}{ }^{\prime}$ is the total private route cost of player role $n$ including tax). In that case, all group members choosing the public route is an equilibrium. However, there might be multiple equilibria and the efficient equilibrium might be unstable. As such, this and other policy possibilities warrant future theoretical and experimental research.

Yet another non-structural way to avert the dilemma is by pre-play communication. Our analysis of the results shows that a few participants did try occasionally to change the course of the game by deviating from equilibrium play. With no pre-play communication, the other group members either ignored these signals, misinterpreted them as errors, or mistrusted them. Pre-play communication in the present setup provides a mechanism to avert the social dilemma by pointing out the long-tern financial benefits to all the group members due to the random assignment of player roles that allows each member to play each of the ten roles with equal probability. 


\section{References}

Anshelevich, E., Dasgupta, A., Kleinberg, J., Tardos, Ė., Wexler, T., \& Roughgarden, T. 2004. The price of stability for network design with fair cost allocation. SIAM Journal of Computing, 38, 1602-1623.

Arnott, E. de Palma, A., \& Lindsey, R. 1990. Economics of a bottleneck. Journal of Urban Economics, 27, 111-130.

Braess, D. 1968. Über ein Paradoxon der Verkehrsplanung. Unternehmensforschung, 12, 258268.

Breitmoser, Y., Tan, J. H. W., \& Zizzo, D. J. 2014. On the beliefs off the path: Equilibrium refinement due to quantal response and level-k. Games and Economic Behavior, 86, 102-125.

Chaudhuri, A. 2011. Sustaining cooperation in laboratory public goods experiments: A selective survey of the literature. Experimental Economics, 14, 47-83.

Croson, R. T. A., \& Marks, M. B. 2000. Step returns in threshold public goods: A meta- and experimental analysis. Experimental Economics, 2, 239-259.

Daniel, T. E., Gisches, E. J., \& Rapoport A. 2009. Departure times in Y-shaped traffic networks with multiple bottlenecks. American Economic Review, 99, 2149-2176.

Dawes, R. 1980. Social dilemmas. Annual Review of Psychology, 31, 169-193.

Denant-Boèmont, L., \& Hammische, S. 2010. Downs-Thomson paradox in cities and endogenous choice of transit capacity: An experimental study. Journal of Intelligent Transport Systems: Technology, Planning, and Operations, 14, 140-153.

Deschenaux, E., Mago, S. D., \& Razzolini, L. 2014. Traffic congestion: An experimental study of the Downs-Thomson paradox. Experimental Economics, 17, 461-487.

Dixit, A. 2003. Clubs with entrapment. American Economic Review, 93, 1824-1829. 
Dixit, V. V., \& Denant-Boemont. 2014. Is equilibrium in transport pure Nash, mixed or stochastic? Transportation Research Part C, 48, 301-310.

Downs, A. 1962. The law of peak-hour expressway congestion. Traffic Quarterly, 16, 393-409.

Fehr, E., \& Schmidt, K. M. 1999. A theory of fairness, competition, and cooperation. Quarterly Journal of Economics, 114, 817-868.

Furuhata, M., Dessouky, M., Ordonez, F., Brunet, M., Wang, X., \& Koenig, S. 2013.

Ridesharing: The state-of-the-art and future directions. Transportation Research Part B: Methodological, 57, 28-46.

Gabuthy, Y., Neveu, M., \& Denant-Boemont, L. 2006. The coordination problem in a structural model of peak-period congestion: An experimental study. Review of Network Economics, 5, 273-298.

Gisches, E. J., \& Rapoport, A. 2012. Degrading network capacity may improve performance: Private vs. public monitoring in the Braess Paradox. Theory and Decision, 73, 267-293. Hartman, J. L. 2012. A route choice experiment with an efficient toll. Networks and Spatial Economics, 12, 205-222.

Iida, Y., Akiyama, T., \& Uchida, T. 1992. Experimental analysis of dynamic route choice behavior. Transportation Research Part B: Methodological, 26, 17-32.

Knorr, F., Chmura, T., \& Schreckenberg, M. 2014. Route choice in the presence of toll road: The role of pre-trip information and learning. Transportation Research Part F: Traffic Psychology and Behaviour, 27, 44-55.

Kocher, M. G., Martinsson, P., Myrseth, K. O. R., \& Wollbrant, C. E. 2017. Strong, bold, and kind: Self-control and cooperation in social dilemmas. Experimental Economics, 20, 44-69. 
Ledyard, J. O. 1995. Public goods: A survey of experimental research. In Handbook of Experimental Economics by J. H. Kagel and A. E. Roth (Eds.). Princeton, NJ: Princeton University Press, 111-194.

Liang, K. Y., \& Zeger, S. L. 1986. Longitudinal data analysis using generalized linear models. Biometrika, 73, 13-22.

Liu, C., Mak, V., \& Rapoport, A. 2015. Cost-sharing in directed networks: Experimental study of equilibrium choice and system dynamics. Journal of Operations Management, 39, 31-47.

Lu, X., Gao, S., Ben-Elia, E., \& Pothering, R. 2014. Traveler' day-to-day route choice behavior with real-time information in a congested risky network. Mathematical Population Studies, $21,205-219$.

Mak, V., Gisches, E. J., \& Rapoport, A. 2015. Route vs. segment: An experiment on real-time travel information in congestible networks. Production and Operations Management, 24, 947-960.

Mak, V., \& Rapoport, A. 2013. The price of anarchy in social dilemmas: Traditional research paradigms and new network applications. Organizational Behavior and Human Decision Processes, 120, 142-153.

McGinty, M., \& Milam, G. 2013. Public goods provision by asymmetric agents: Experimental evidence. Social Choice and Welfare, 40, 1159-1177.

Messick, D., \& Brewer, M. 1983. Solving social dilemmas: A review. Review of Personality and Social Psychology, 4, 11-44.

Morgan, J., Orzen, H., \& Sefton, M. 2009. Network architecture and traffic flows: Experiments on the Pigou-Knight-Downs and Braess paradoxes. Games and Economic Behavior, 66, 348372. 
Moulin, H. J. 2003. Fair Division and Collective Welfare. Cambridge, MA: The MIT Press.

Nisan, N., Roughgarden, T., Tardos, Ė., \& Vazirani, V. V. (Eds). 2007. Algorithmic Game Theory. Cambridge, MA: Cambridge University Press.

Pigou, A. C. 1920. The Economics of Welfare. London: MacMillan.

Rapoport, A., Kugler, T., Dugar, S., \& Gisches, E. J. 2009. Choice of routes in congested traffic networks: experimental tests of the Braess paradox. Games and Economic Behavior, 65, 538571.

Rapoport, A., Gisches, E. J., \& Mak, V. 2014. Distributed decisions in networks: Laboratory study of routing splittable flow. Production and Operations Management, 23, 314-331.

Rapoport, A., Mak, V., \& Zwick, R. 2006. Navigating congested networks with variable demand: Experimental evidence. Journal of Economic Psychology, 27, 648-666.

Roughgarden, T. 2005. Selfish Routing and the Price of Anarchy. Cambridge, MA: MIT Press.

Schelling, T. C. 1978. Micromotives and Macrobehavior. New York: W. W. Norton \& Company.

Selten, R., Chmura, T., Pitz, T., \& Schreckenberg, M. 2007. Commuters' route choice behavior. Games and Economic Behavior, 58, 394-406.

Thomson, A. 1977. Great Cities and Their Traffic. London: Gollancz.

Van Huyck, J., Battalio, R., \& Beil, R. 1990. Tacit coordination games, strategic uncertainty, and coordination failure. American Economic Review, 80, 234-248.

Van Huyck, J., Battalio, R., \& Beil, R. 1991. Strategic uncertainty, equilibrium selection principles, and coordination failure in average opinion games. Quarterly Journal of Economics, 106, 885-911. 
Van Lange, P. A. M., Joireman, J., Parks, C. D., \& Van Dijk, E. 2013. The psychology of social dilemmas: A review. Organizational Behavior and Human Decision Processes, 120, 125-141. Wijayaratna, K. P., Dixit, V. V., Denant-Boemont, L. D., \& Waller, S. T. 2017. An experimental study of the Online Information Paradox: Does en-route information improve road network performance? PLoS ONE, 12: e018149.

Zelmer, J. 2003. Linear public goods experiments: A meta-analysis. Experimental Economics, 6, 299-310. 
Table 1 Characteristics of the private and public routes

(a) The Private Route Costs and Associated Critical Masses by Condition

\begin{tabular}{cccccccccccc}
\hline Player $(j)$ & $1^{\mathrm{H}}$ & $2^{\mathrm{H}}$ & $3^{\mathrm{H}}$ & $4^{\mathrm{H}}$ & $5^{\mathrm{H}}$ & $6^{\mathrm{HL}}$ & $7^{\mathrm{HL}}$ & $8^{\mathrm{HL}}$ & $9^{\mathrm{HL}}$ & $10^{\mathrm{HL}}$ & Total \\
\hline Private route cost $=c_{j}$ & 93 & 43 & 26 & 18 & 13 & 9 & 7 & 5 & 4 & 3 & 221 \\
\hline $\begin{array}{c}\text { Critical mass } \\
\text { Condition H: }\end{array}$ & 1 & 2 & 4 & 6 & 8 & Nil & Nil & Nil & Nil & Nil \\
$\begin{array}{c}\text { Public route cost }=120 / i \\
\text { Condition L: }\end{array}$ & 0 & 1 & 2 & 3 & 4 & 6 & 7 & Nil & Nil & Nil \\
Public route cost $=60 / i$ & & & & & & & & & & & \\
\hline
\end{tabular}

(b) The Public Route Costs

\begin{tabular}{cccccccccccc}
\hline $\begin{array}{c}\text { No. of players choosing } \\
\text { the public route }(i)\end{array}$ & 1 & 2 & 3 & 4 & 5 & 6 & 7 & 8 & 9 & 10 & Total \\
\hline $\begin{array}{c}\text { Condition H: } \\
\text { Public route cost }=120 / i\end{array}$ & 120 & 60 & 40 & 30 & 24 & 20 & 17 & 15 & 13 & 12 & 120 \\
$\quad \begin{array}{c}\text { Condition L: } \\
\text { Public route cost }=60 / i\end{array}$ & 60 & 30 & 20 & 15 & 12 & 10 & 8 & 7 & 6 & 6 & 60 \\
\hline
\end{tabular}

Notes: The top panel displays the travel costs on the 10 private routes. It also lists the associated critical masses by player role and condition. For each player indexed by role, the critical mass in a condition is the minimum number of players other than that player who have chosen the public route in order for the public route to be at least as preferred as the private route. "Nil" entries mean that a critical mass does not exist for the associated player in the context of the game, as the private route strictly dominates the public route for that player. In addition, in the top panel, the superscripts $\mathrm{H}$ and $\mathrm{L}$ in the first row indicate whether the corresponding player chooses the private route in equilibrium in Conditions $\mathrm{H}$ and $\mathrm{L}$, respectively. The bottom panel lists the costs on the single public route dependent on the number of players on it by condition. 
Table 2 Mean choice frequencies of the public route by condition, group, and block (20 rounds each) with the session overall mean travel costs per player, the equilibrium predictions (“Eq."), and the social optima

(a) Condition $\mathrm{H}$

\begin{tabular}{|c|c|c|c|c|c|c|c|c|c|c|c|c|c|c|}
\hline & Round & H1 & $\mathbf{H} 2$ & H3 & H4 & H5 & H6 & $\mathbf{H 7}$ & H8 & H9 & H10 & $\begin{array}{c}\text { Block } \\
\text { overall }\end{array}$ & Eq. & $\begin{array}{c}\text { Social } \\
\text { optimum }\end{array}$ \\
\hline \multirow{5}{*}{$\begin{array}{l}\text { Mean } \\
\text { no. of } \\
\text { players } \\
\text { choosing } \\
\text { public } \\
\text { route }\end{array}$} & $1-20$ & 0.60 & 0.80 & 1.65 & 1.20 & 1.40 & 0.25 & 0.80 & 1.05 & 0.35 & 2.10 & $1.02(0.58)$ & 0 & 10 \\
\hline & $21-40$ & 0.05 & 0.05 & 0.50 & 0.35 & 0.55 & 0.00 & 0.00 & 0.10 & 0.05 & 1.25 & $0.29 * *(0.40)$ & 0 & 10 \\
\hline & $41-60$ & 0.00 & 0.00 & 0.00 & 0.10 & 0.00 & 0.00 & 0.00 & 0.00 & 0.00 & 1.55 & $0.17(0.49)$ & 0 & 10 \\
\hline & $61-80$ & 0.00 & 0.05 & 0.00 & 0.00 & 0.00 & 0.00 & 0.00 & 0.00 & 0.00 & 0.70 & $0.08(0.22)$ & 0 & 10 \\
\hline & $\begin{array}{c}\text { Session } \\
\text { overall }\end{array}$ & 0.16 & 0.23 & 0.54 & 0.41 & 0.49 & 0.06 & 0.20 & 0.29 & 0.10 & 1.40 & $0.39(0.39)$ & $\mathbf{0}$ & 10 \\
\hline \multicolumn{2}{|c|}{$\begin{array}{c}\text { Mean travel cost } \\
\text { per player } \\
\text { (session overall) }\end{array}$} & 22.83 & 22.73 & 23.13 & 23.26 & 23.03 & 22.29 & 22.95 & 22.52 & 22.60 & 24.05 & $22.94(0.49)$ & 22.1 & 12 \\
\hline
\end{tabular}


(b) Condition L

\begin{tabular}{|c|c|c|c|c|c|c|c|c|c|c|c|c|c|c|}
\hline & Round & $\mathbf{L} 1$ & $\mathbf{L 2}$ & L3 & L4 & L5 & L6 & L7 & L8 & L9 & L10 & $\begin{array}{c}\text { Block } \\
\text { overall } \\
\end{array}$ & Eq. & $\begin{array}{c}\text { Social } \\
\text { optimum }\end{array}$ \\
\hline \multirow{5}{*}{$\begin{array}{l}\text { Mean } \\
\text { no. of } \\
\text { players } \\
\text { choosing } \\
\text { public } \\
\text { route }\end{array}$} & $1-20$ & 4.60 & 6.30 & 4.05 & 4.90 & 3.95 & 4.90 & 5.10 & 4.80 & 5.05 & 5.65 & $4.93(0.69)$ & 5 & 10 \\
\hline & $21-40$ & 5.05 & 6.20 & 4.30 & 6.20 & 5.25 & 5.35 & 6.30 & 4.95 & 5.40 & 5.30 & $5.43 *(0.64)$ & 5 & 10 \\
\hline & $41-60$ & 4.90 & 6.15 & 4.40 & 5.90 & 5.75 & 5.05 & 6.10 & 5.60 & 5.45 & 6.05 & $5.53(0.59)$ & 5 & 10 \\
\hline & $61-80$ & 4.75 & 6.15 & 4.65 & 6.30 & 5.65 & 5.00 & 6.50 & 5.55 & 5.55 & 5.20 & $5.53(0.64)$ & 5 & 10 \\
\hline & $\begin{array}{c}\text { Session } \\
\text { overall }\end{array}$ & 4.83 & 6.20 & 4.35 & 5.83 & 5.15 & 5.08 & 6.00 & 5.23 & 5.36 & 5.55 & $5.36(0.56)$ & 5 & 10 \\
\hline \multicolumn{2}{|c|}{$\begin{array}{l}\text { Mean travel cost } \\
\text { per player } \\
\text { (session overall) }\end{array}$} & 9.34 & 8.13 & 11.28 & 8.39 & 9.06 & 8.96 & 8.48 & 9.43 & 10.20 & 8.51 & $9.18(0.96)$ & 8.8 & 6 \\
\hline
\end{tabular}

Notes: Each group in Conditions H and L is denoted as H1-H10 and L1-L10, respectively. The "Block overall" column includes standard deviations in parentheses with group as the unit of analysis. Where the observed mean choice frequencies of the public route between every pair of successive blocks are significantly different according to a pairwise $t$-test, the block overall entry for the later block is marked by one or more asterisks $(* p<0.05, * * p<0.01)$. 
Table 3 GEE regression results of individual travel cost on number of deviations from equilibrium

\begin{tabular}{ccccc}
\hline & Condition H & Condition L & \multicolumn{2}{c}{ Condition L } \\
& & (All Roles) & Roles 1-5 & Roles 6-10 \\
\hline \# Observations & 8,000 & 8,000 & 4,000 & 4,000 \\
\hline $\begin{array}{c}\text { Intercept } \\
\text { \# Deviations } \\
\text { from equilibrium }\end{array}$ & $1,752.9(13.8)^{* *}$ & $660.2(8.93)^{* *}$ & $430.1(8.86)^{* *}$ & $221.9(1.01)^{* *}$ \\
\hline QIC & $26.47(1.72)^{* *}$ & $8.17(1.95)^{*}$ & $20.25(2.56)^{* *}$ & $3.27(0.35)^{* *}$ \\
\hline
\end{tabular}

Notes: Each entry in rows 3-4 is the regression coefficient of the corresponding independent variable with standard error in parentheses; where an estimate is significantly different from zero, it is marked by one or more asterisks $(* p<0.05$; ** $p<0.01)$. QIC is the goodness-of-fit statistic for GEE. 
Fig. 1 A directed network representation for the model

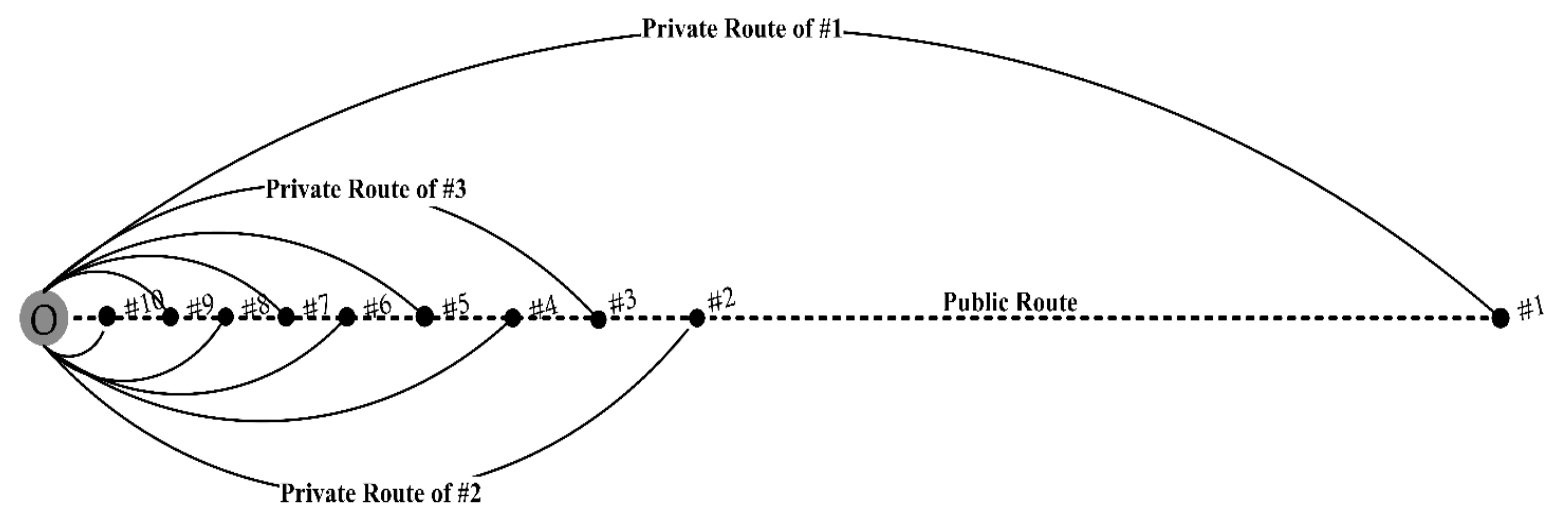

Notes:

(1) Distances on the horizontal line are proportional to the travel costs on the private route.

(2) To save space, we do not index the private routes except for routes 1, 2, and 3.

(3) The experimental instructions have a different representation, but with equivalent strategic interactions and social dilemmas. 
Fig. 2 Mean choice frequencies of the public route by condition and round
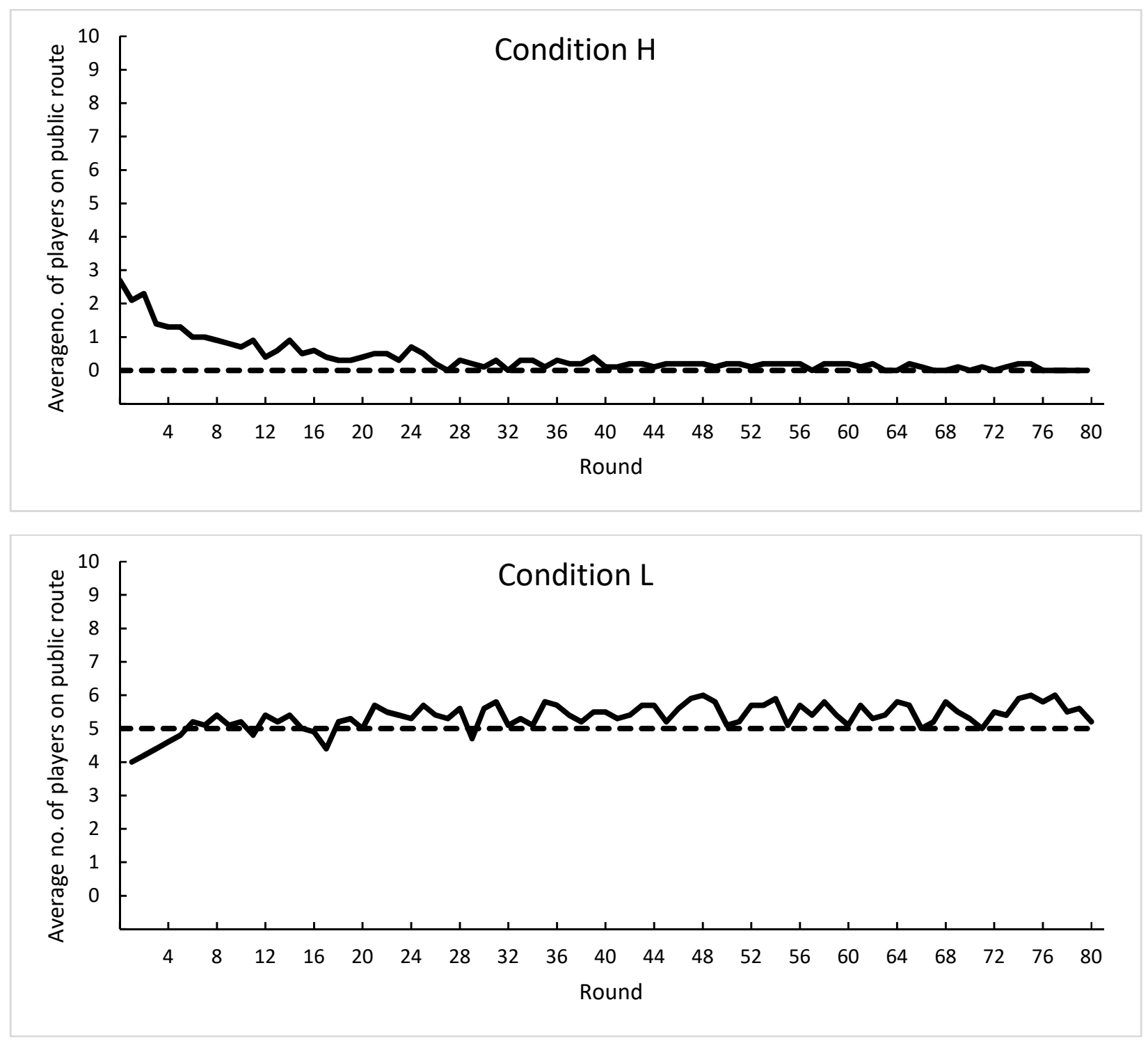

Note: The dotted line in every panel indicates the Nash equilibrium solution: no player choosing the public route in Condition $\mathrm{H}$, and five players choosing that route in Condition $\mathrm{L}$. 
Fig. 3 Number of deviations from equilibrium by condition and player role in the first and second half of the experimental session
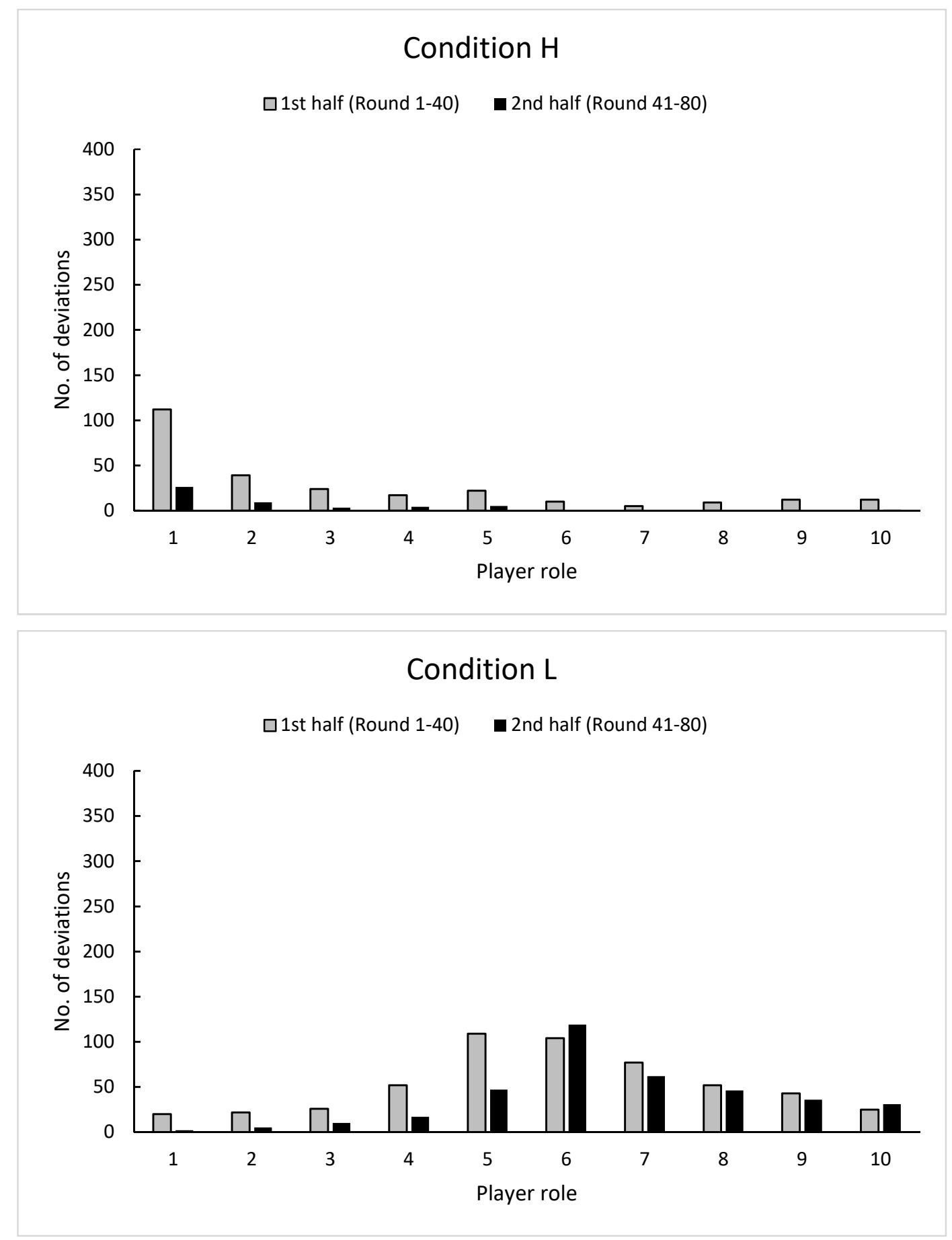

Note: Each bar indicates the total number of deviations out of $400(=40$ rounds per half of a session $\times 10$ groups) data points. 


\section{When a Few Undermine the Whole: \\ A Class of Social Dilemmas in Ridesharing}

\section{Online Appendix A: Sample Instructions to Participants (Condition L)}

\section{$\underline{\text { Introduction }}$}

Welcome to this laboratory experiment on route choice in traffic networks. During this experiment, you will be asked to make a large number of decisions and so will the other participants. Your decisions, as well as the decisions of the other participants, will determine your monetary payoff according to the rules of the game that will be explained shortly.

Please read the instructions carefully. If you have any questions, please raise your hand and one of the supervisors will come to assist you.

Note that hereafter communication between the participants is prohibited. If they communicate with one another in any shape or form, then the experiment will be terminated.

\section{$\underline{\text { The Route Selection Task }}$}

The experiment is computerized. You will make your decisions by clicking on the appropriate buttons that will appear on your screen (please do not use the keyboard).

There are $\mathbf{1 0}$ participants in the lab, who will play together as a group for the entire session. On each round of the experiment, each participant will be numbered randomly from 1 through 10 .

In this experiment, you will participate in $\mathbf{8 0}$ identical rounds of a network routing game. On each round, you will be presented with a diagram of a traffic network with a single origin, a single destination, and $\mathbf{1 1}$ different routes connecting them. Your task, as well as the task of all other participants, is to choose one of two routes to traverse: the first is a private route that only you can choose, and the other is a common pooled route that all other participants may choose as well.

\section{Description of the Game}


Consider the traffic network that is exhibited in the diagram below. You are required to choose a route that connects the origin, denoted by the letter $\mathbf{O}$ (for Origin), to the final destination denoted by the letter D (for Destination).

\section{(Please study this diagram.)}

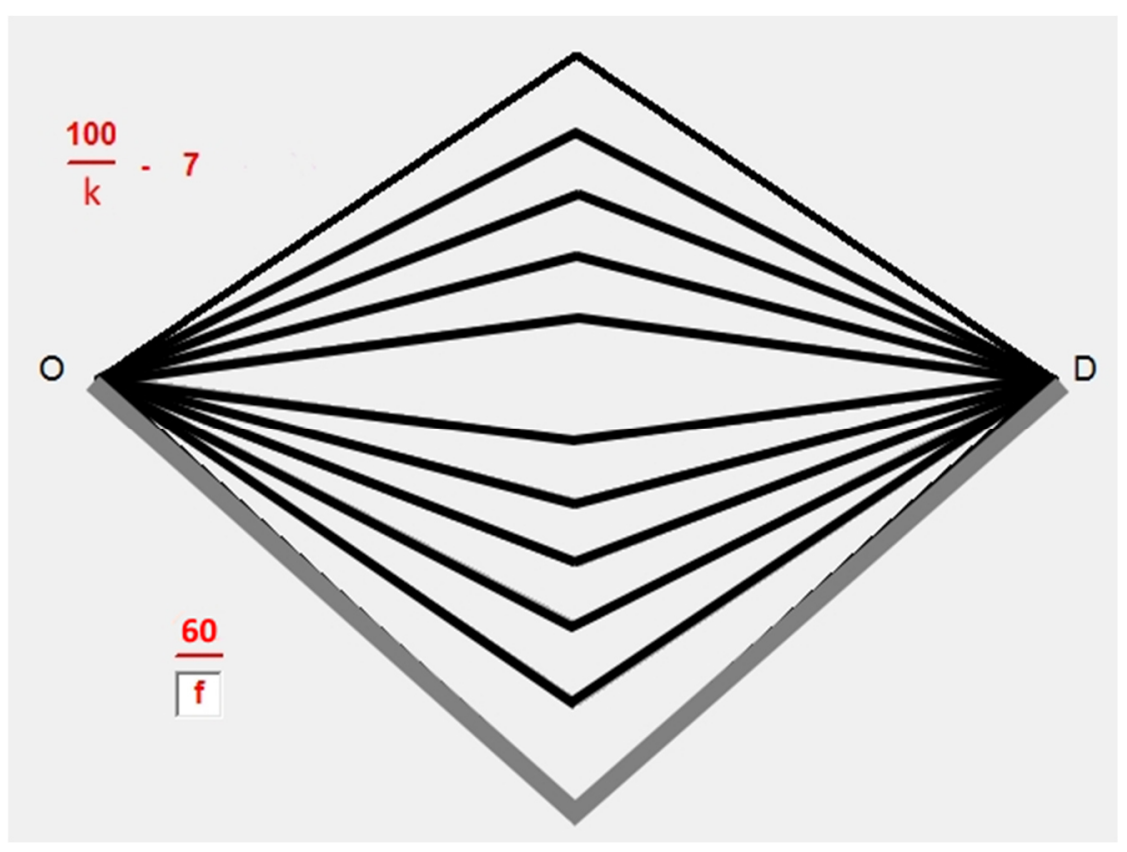

Traversing a network is always costly in terms of the time needed to complete each segment of the road, gas, tolls, etc. The above network has two types of routes; 10 private routes (top ten) and 1 pooled (bottom one). The travel costs are shown near the routes. For the private routes, they differ from one participant to another and are computed by the equation $\frac{100}{k}-7$, where $k$ indicates the participant number (from 1 to 10 ). For example, for participant \#1 (top route) the cost is $\frac{100}{1}-7=93$, for participant $\# 2$ (second from top) it is $\frac{100}{2}-7=43$, and for participant $\# 10$ (second from bottom) it is $\frac{100}{10}-7=3$.

The cost of the common pooled route is the same for all the participants choosing it, and it depends on their number. For example, if only a single participant chooses it, then the cost for her would be $\frac{60}{1}=60$. If two participants choose the pooled road, then the cost for each of them would be $\frac{60}{2}=30$, and if all 10 participants choose it, then travel cost for each participant would be $\frac{60}{10}=6$. 
At the end of each round, you will receive a reward of $\mathbf{5 0}$ for reaching the destination. Your earnings will be determined by subtracting your cost for the round from this reward. For example, if you are

participant $\# 5$ and you chose the private road, then your cost on that round would be $\quad \frac{100}{5}-7=17$ and your payoff would be $50-\left(\frac{100}{5}-7\right)=50-17=33$.

All the participants choose routes simultaneously and independently of one another, leaving the origin $\mathbf{O}$ and traveling to the destination $\mathbf{D}$ without knowing the other participants' decisions.

\section{Example:}

Suppose that on a certain round you were assigned to be participant $\# 1$ and decided to choose your private route (top route), which is indicated below in blue.

Suppose that participants \#3, \#5, \#6, \#9, \#10 also chose their private routes as indicated in green.

Finally, suppose that the remaining participants \#2, \#4, \#7 and \#8 chose the common pooled route (their private route remained black).

At the end of such a round, the computer will display the results as in the screen below: 


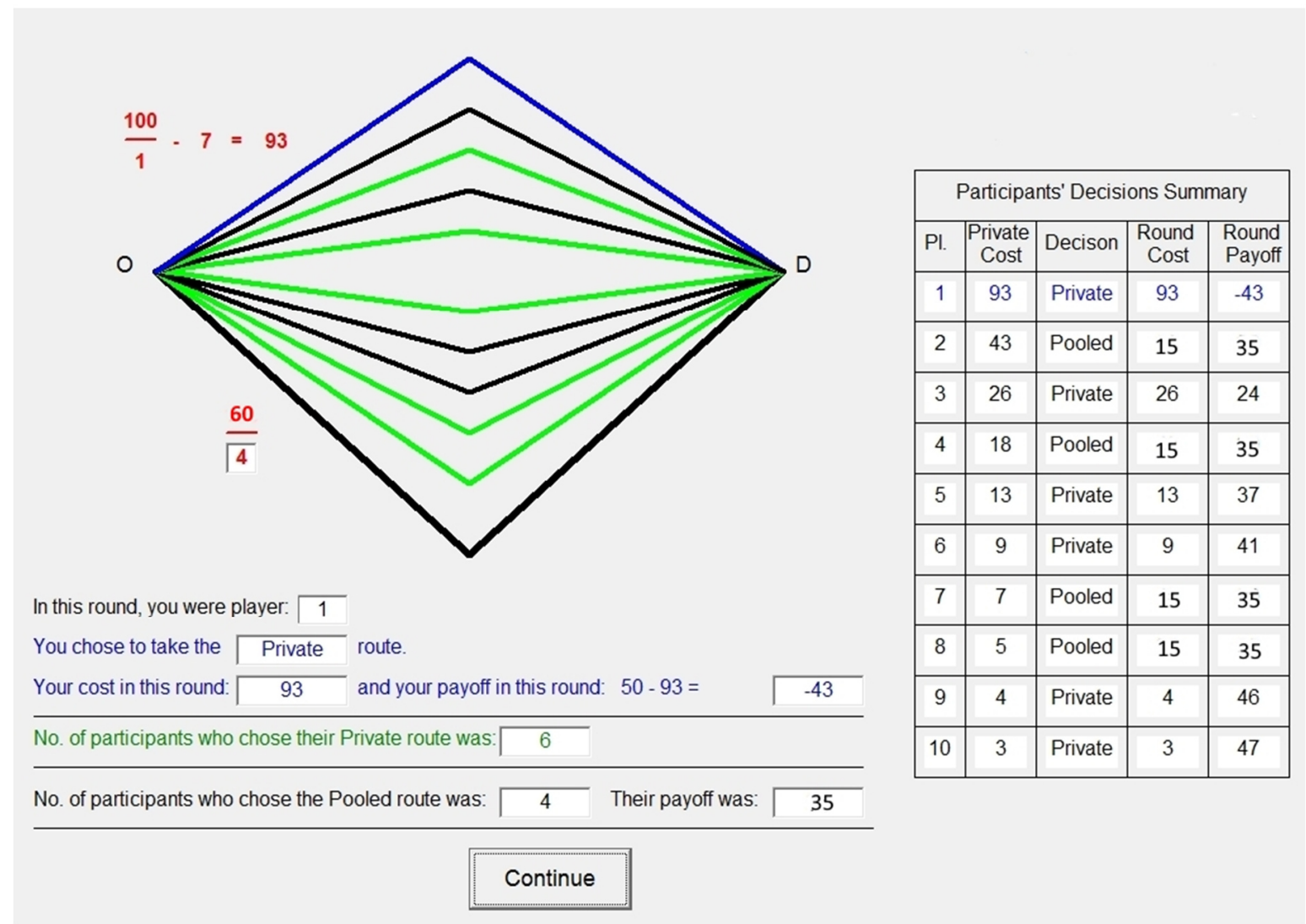

The top part of the screen exhibits the network and the routes chosen by the 10 participants. The route that you chose is marked in blue (route \#1). Private routes chosen by other participants are marked in green (routes $\# 3, \# 5, \# 6, \# 9$, and $\# 10)$. Routes that remained black $(\# 2, \# 4, \# 7$, and $\# 8$ ) were not chosen by their respective participants, and hence those participants chose the pooled route.

The bottom part of the screen displays your number for the present round of play (row 1); your choice on this round (row 2); your cost and your payoff (row 3); the number of participants who chose their private route (row 4); and in the last row the number of participants who chose the pooled route and their payoff.

The table on the right gives more detail presenting the ten participants ( $1^{\text {st }}$ column), the cost of their private routes $\left(2^{\text {nd }}\right.$ column $)$, their choice $\left(3^{\text {rd }}\right.$ column $)$, the resulting cost $\left(4^{\text {th }}\right.$ column $)$ and payoff $\left(5^{\text {th }}\right.$ column).

In this example, the payoff that you (participant \#1) earned by choosing your private route:

Travel cost: $\frac{100}{1}-7=93$ 
Earning: $50-\left(\frac{100}{1}-7\right)=50-93=-43$

For the 5 participants who also chose the private route:

Travel cost: 26, 13, 9, 4, 3 for player \#3, \#5, \#6, \#9, and \#10, respectively.

Earnings: 24, 37, 41, 46, 47, respectively.

And for the 4 participants who chose the common pooled route:

Travel cost: $\frac{60}{4}=15$

Individual earnings: $50-\frac{60}{4}=50-15=35$

Note that the payoff on the pooled route and some of the private routes may be negative.

You will also be provided with a History screen (see example below) exhibiting your private route and payoff as well as the number of participants choosing the common pooled route and the associated payoff in all previous rounds. Your decision on each round is indicated in blue. If you did not choose your private route or no participant selected the common pooled route, then your payoff will be indicated as N/A. You may refer to the History screen at any time during the session.

\begin{tabular}{|l|c|c|c|c|c|}
\hline \multicolumn{4}{|c|}{$\Delta$} & & \multicolumn{2}{|c|}{ Your Private Route } & \multicolumn{2}{c|}{ Pooled Route } \\
\cline { 2 - 6 } & Round & Route & Payoff & \# Players & Payoff \\
\cline { 2 - 6 } & 1 & 1 & -43 & 4 & 35 \\
\hline & & & & \\
\hline & & & & \\
\hline & & & & \\
\hline & & & & \\
\hline & & & & & \\
\hline & & & & & \\
\hline
\end{tabular}

All 80 rounds will have exactly the same structure. 


\section{Procedure}

At the beginning of each round, the computer will randomly assign you a role (i.e., your role will most likely change on each round) and display a network diagram similar to the one exhibited before. You will then be asked to choose either your private route or the common pooled route. To choose a route, simply use the mouse to click on it. The color of the route you choose will change to blue indicating your choice. To change your choice, please click on the route again and its color will change back to black. Once you are satisfied with your selection, please press the "Confirm" button. You will be asked to verify your choice.

Once all the 10 participants confirm their choice of routes, the computer will automatically exhibit a feedback screen. After studying the summary information about the last round, you can click the "continue" button and proceed to the next round.

\section{$\underline{\text { Payments }}$}

At the end of the experiment, you will be paid for your cumulative earnings in all the 80 rounds. You will be paid in cash for your earnings with an exchange rate of $\$ 1=110$ tokens. In addition, you will receive a show up bonus of $\$ 5$ for attending the experiment.

Once you are certain that you understand the task, please place the instructions on the table in front of you to indicate that you have completed reading them. If you have any questions, please raise your hand and one of the supervisors will come to assist you.

The experiment will begin shortly. Thank you for your participation. 


\section{Online Appendix B: Additional Figures}

Fig. A.1. The choice frequencies of the public route by group and round in Condition $\mathrm{H}$ (equilibrium prediction marked by dotted line in every panel).
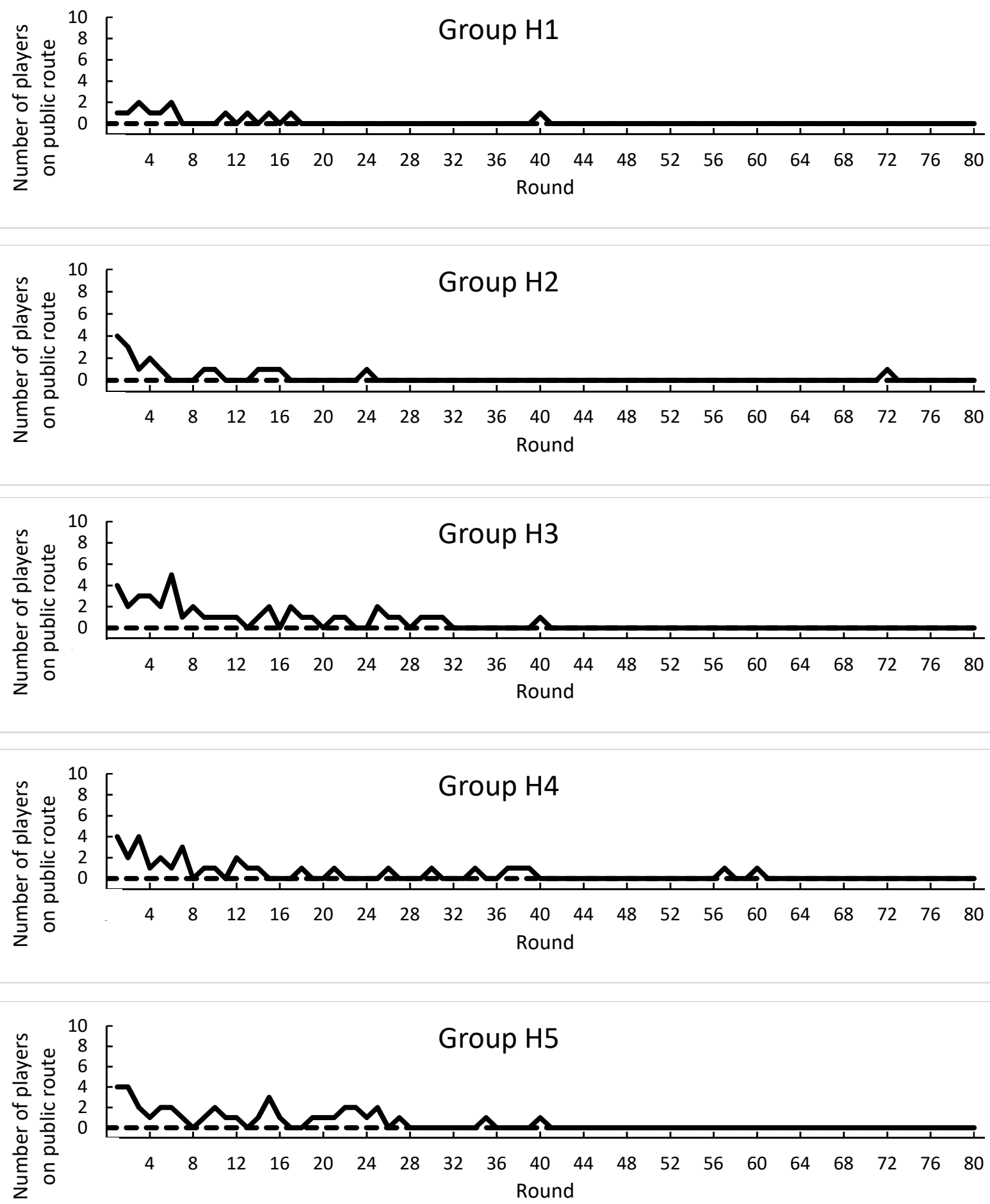
Fig. A.1. (cont'd)
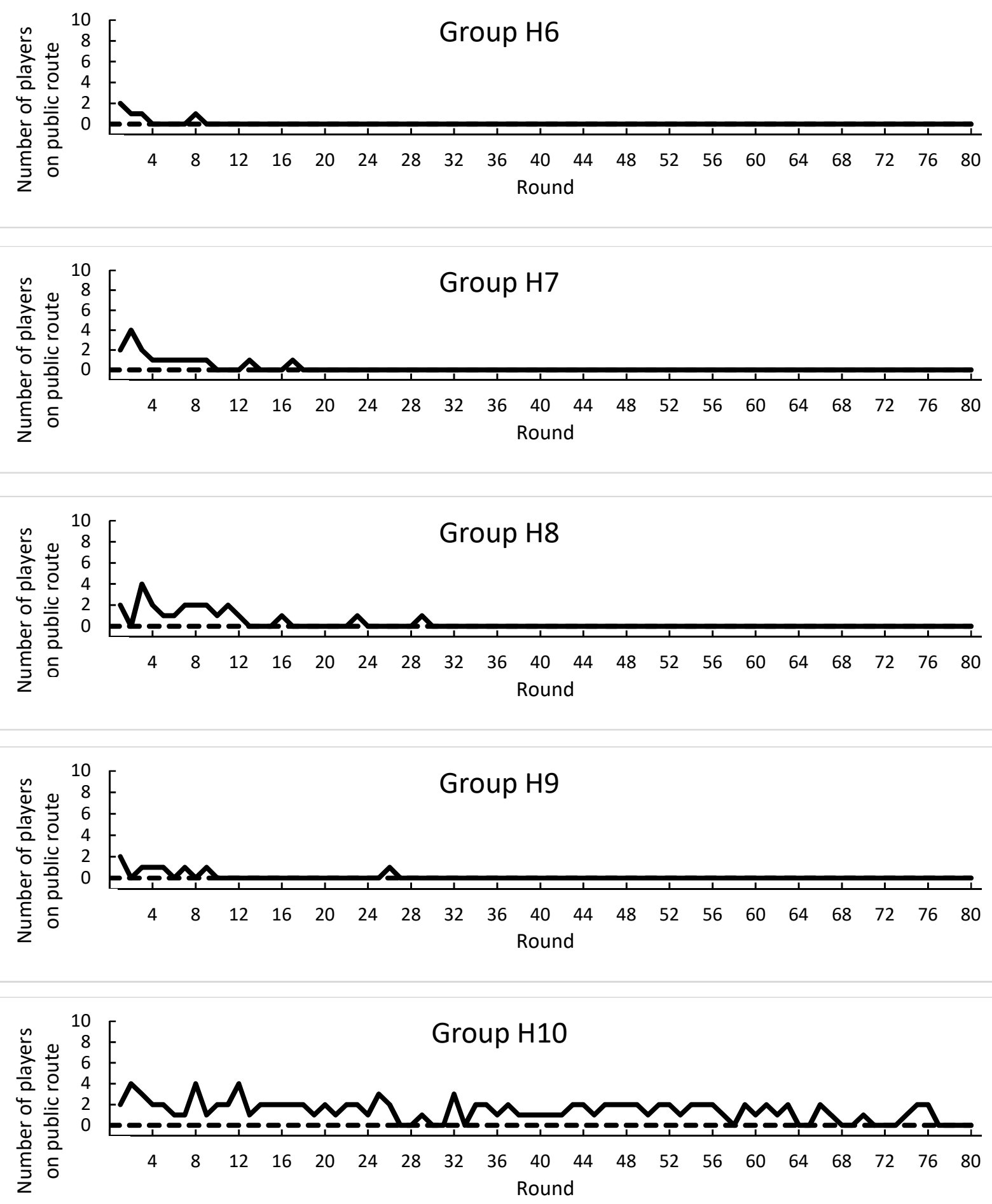
Fig. A.2. The choice frequencies of the public route by group and round in Condition L (equilibrium prediction marked by dotted line in every panel).
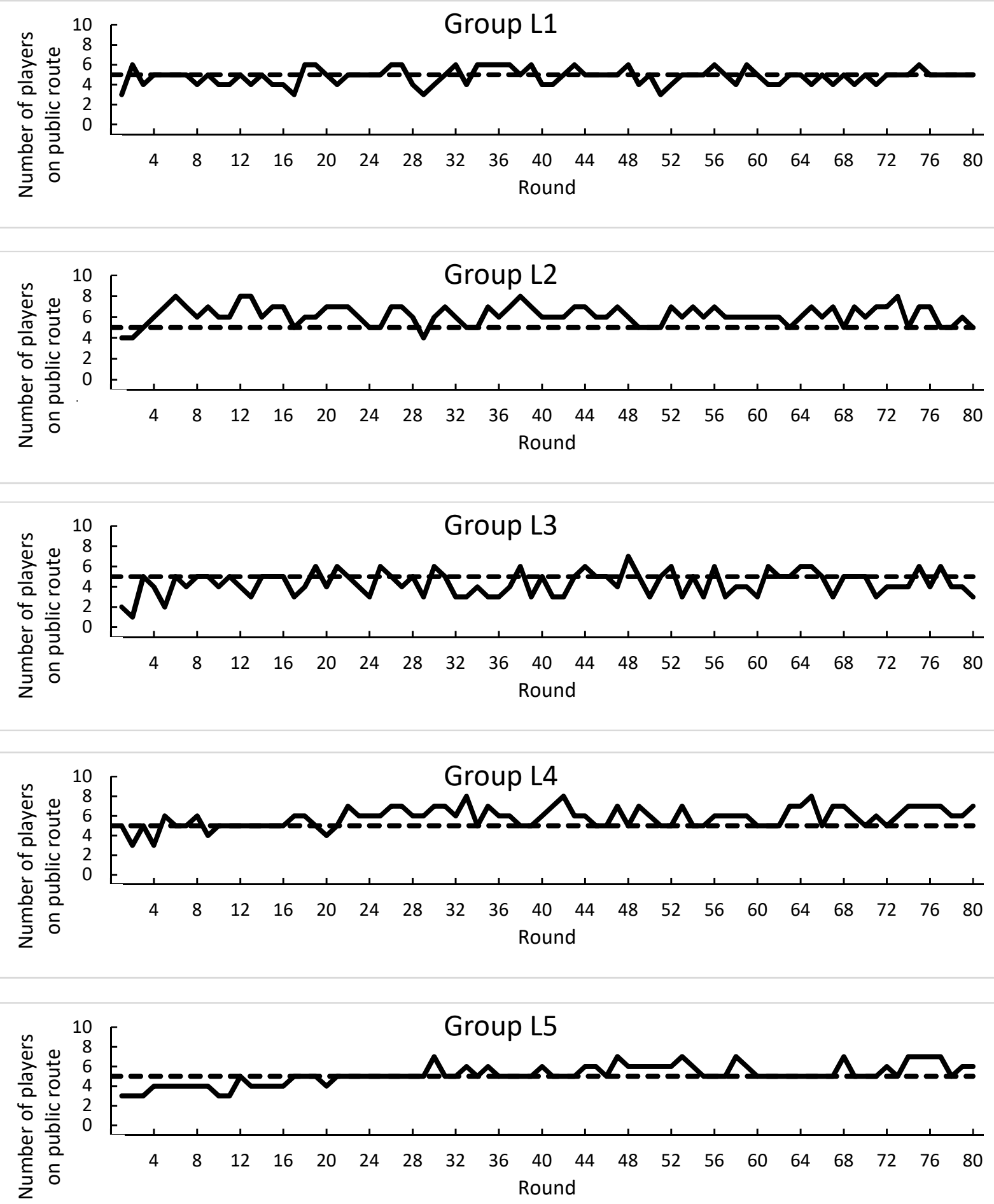
Fig. A.2. (cont'd)
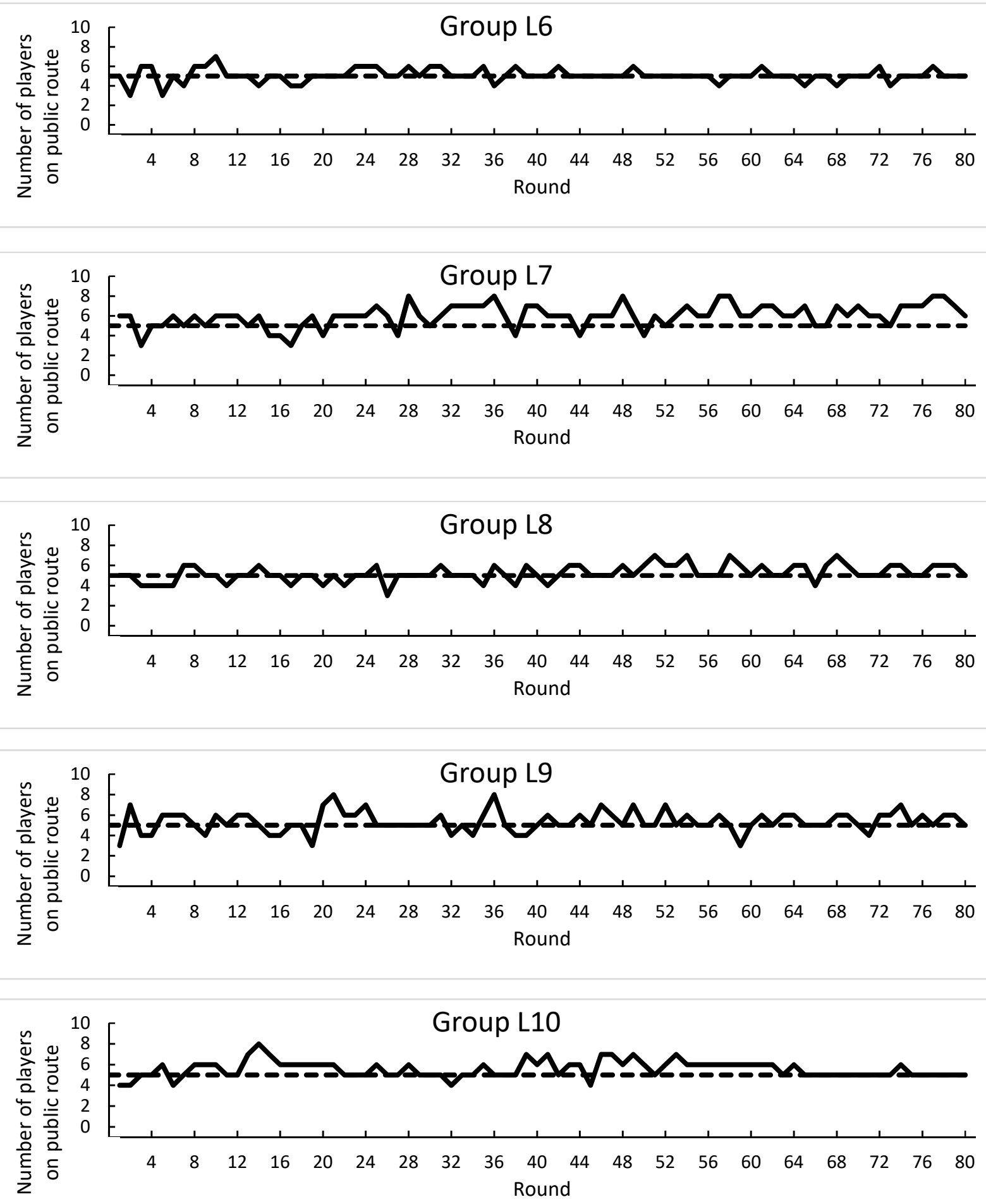
Fig. A.3. Box-and-whisker plots showing the distribution of individual players' total number of deviations from equilibrium throughout the session, by condition and group.
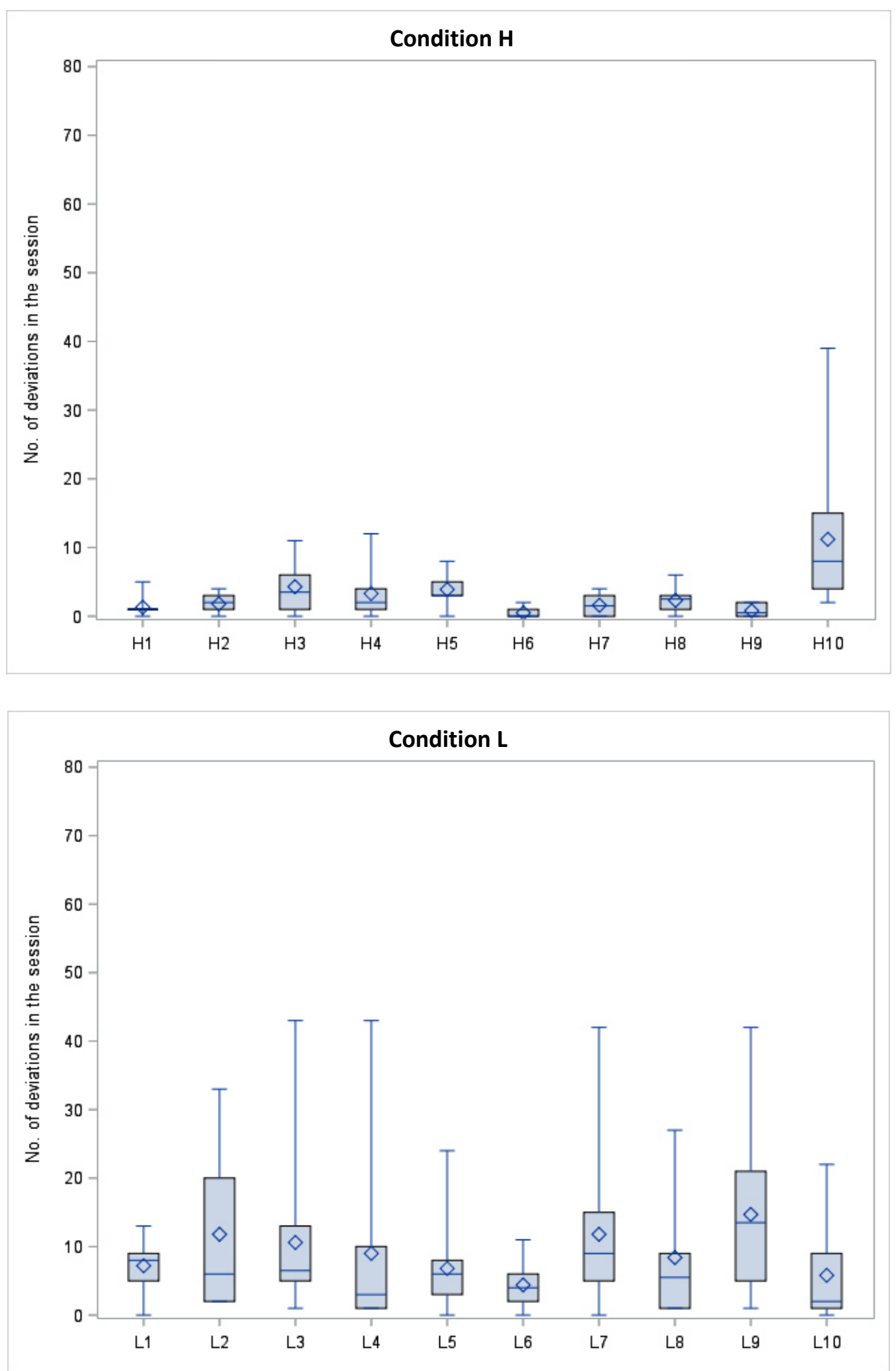